\title{
Thermal squeezeout of dark matter
}

\author{
Pouya Asadi, ${ }^{1}$ Eric David Kramer, ${ }^{2}$ Eric Kuflik, ${ }^{2}$ Gregory W. Ridgway, ${ }^{1}$ Tracy R. Slatyer, ${ }^{1}$ and Juri Smirnov ${ }^{3,4}$ \\ ${ }^{1}$ Center for Theoretical Physics, Massachusetts Institute of Technology, \\ Cambridge, Massachusetts 02139, USA \\ ${ }^{2}$ Racah Institute of Physics, Hebrew University of Jerusalem, Jerusalem 91904, Israel \\ ${ }^{3}$ Center for Cosmology and AstroParticle Physics (CCAPP), The Ohio State University, \\ Columbus, Ohio 43210, USA \\ ${ }^{4}$ Department of Physics, The Ohio State University, Columbus, Ohio 43210, USA
}

(Received 26 April 2021; accepted 9 October 2021; published 15 November 2021)

\begin{abstract}
We carry out a detailed study of the confinement phase transition in a dark sector with an $S U(N)$ gauge group and a single generation of a dark heavy quark. We focus on heavy enough quarks such that their abundance freezes out before the phase transition, and the phase transition is of first order. We find that during this phase transition, the quarks are trapped inside contracting pockets of the deconfined phase and are compressed enough to interact at a significant rate, giving rise to a second stage of annihilation that can dramatically change the resulting dark matter abundance. As a result, the dark matter can be heavier than the often-quoted unitarity bound of $\sim 100 \mathrm{TeV}$. Our findings are almost completely independent of the details of the portal between the dark sector and the Standard Model. We comment briefly on possible signals of such a sector. Our main findings are summarized in a companion paper, while here we provide further details on different parts of the calculation.
\end{abstract}

DOI: 10.1103/PhysRevD.104.095013

\section{INTRODUCTION}

The cosmic abundance of dark matter (DM) is comparable to the abundance of Standard Model (SM) particles up to an $\mathcal{O}(1)$ factor [1]. The similarity of these two ostensibly unrelated abundances raises the suspicion that the two sectors may have been in chemical equilibrium at some point in their history, implying some sort of interaction portal between the SM and DM. Numerous experimental efforts to look for such a portal are underway. Nonetheless, the particle nature of DM and any potential portals to the SM remain unknown at present.

In addition to probing interactions between DM and the SM, many experimental and theoretical efforts aim to probe possible dynamics within the dark sector itself. Often simplified dark sectors with only a single DM particle are considered. Yet, the rich gauge structure of the SM offers no particular reason to believe that the dark sector will be significantly simpler. A wide range of more involved dark sectors have been studied, especially scenarios with a new confining force in the dark sector; see for instance Refs. [2-35]. Depending on the details of the sector,

Published by the American Physical Society under the terms of the Creative Commons Attribution 4.0 International license. Further distribution of this work must maintain attribution to the author(s) and the published article's title, journal citation, and DOI. Funded by SCOAP ${ }^{3}$. different hadronic states can be the DM candidate in different theories, and the stabilizing symmetry and the DM mass scale can vary widely [30]. Such a sector can further give rise to rich dynamics that can potentially solve other problems in the SM as well; e.g., see Refs. [6,36] where the observed baryon asymmetry is tied to the DM abundance.

An interesting class of confining dark sector models is the scenario where all the dark quarks are substantially heavier than the dark confinement scale $\Lambda$. These models and their experimental signals have been studied extensively; see for instance Refs. [6,24-26,28]. For sufficiently heavy quarks, lattice calculations have shown that the phase transition in such a sector is of first order for $S U(N)$ with $N=3$ [37-41] or $N>3[42,43]$. There has been a recent surge of interest in the study of the potential effects of firstorder phase transitions in other DM models; e.g., see Refs. [44-47], ${ }^{1}$ but the effects of the phase transition on the relic abundance of dark matter in confining dark sector models are mostly unexplored, with the exception of a recent study of dark sectors with only light quarks $\left(m_{q} \leq \Lambda\right)[51]$.

In this work, we consider the simplest such confining model-an $S U(3)$ gauge theory with one heavy quark in

\footnotetext{
${ }^{1}$ See also Refs. [48-50] for another mechanism affecting DM abundance in the presence of significant supercooling during a phase transition.
} 
the fundamental representation - and focus on the effects of the first-order phase transition on the DM relic abundance calculation. Similar to the arguments put forward in Ref. [52], we will argue that toward the end of the phase transition, we will be left with pockets of the high temperature (i.e., deconfined) phase submerged in a sea of low temperature (i.e., confined) phase. We will argue that the heavy quarks are all initially trapped inside these contracting pockets. ${ }^{2}$ To determine important properties of these pockets such as their initial characteristic size and contraction rate, we will develop a simplified model to numerically simulate the phase transition.

As a pocket contracts, the dark quarks within it are compressed, allowing them to recouple and go through a second stage of annihilation. We calculate the fraction of the quarks that survive this new annihilation stage and escape the pockets in the form of dark baryons. We refer to this process as "thermal squeezeout," as the dark quarks are squeezed within the pockets and eventually leak out, in contrast to the standard "thermal freezeout." We find a dramatic suppression in the final abundance thanks to this phenomenon, which points to much heavier dark matter parameter space than was previously thought. In particular, the fact that the local DM density is much larger than the globally averaged DM density during this second stage of annihilation invalidates the homogeneity assumption made in the unitarity bound argument of Ref. [53]. ${ }^{3}$ As a result, this model allows the DM candidate to be heavier than the perturbative unitarity bound on weakly interacting massive particles (WIMPs), despite being thermal.

Since this second stage of annihilation is controlled by the dynamics within the dark sector and not the interaction between the dark sector and SM, our results are largely independent of the portal to the SM. In fact, we do not constrain ourselves to any specific portal in this paper. The only assumptions we make about such a portal are that (i) it exists, (ii) it keeps the SM and the dark sector in thermal contact during the phase transition, and (iii) it respects the dark baryon number that stabilizes the dark baryons. These assumptions streamline our calculations significantly. However, it is worth considering the possibility of models in which we can relax one or more of these assumptions; we leave this for future work.

\footnotetext{
${ }^{2}$ This depends on the representation of the quarks under the dark confining gauge group. For instance, if the quarks are in the adjoint representation (similar to the model in Ref. [27]), they could combine with the surrounding gluons, form color-neutral hadrons, and move into the confined phase. Similarly, if in addition to the heavy quarks the spectrum were to include light quarks in the fundamental representation as well, the heavy quarks would not remain trapped within the pocket as they could make a color-neutral bound state with one of many light quarks surrounding them to escape the pocket.

${ }^{3}$ See also Refs. [54,55] for more recent studies of the unitarity bound on thermal DM models.
}

The current work is merely the start of a broader program of studying such models in more details. The phenomenology of all such models should be revisited in light of the dramatic change in the relic abundance calculation. Depending on the gauge group under study, the quarks' representation, and the portal, different models (with vastly different phenomenology) can be constructed.

Our study indicates a natural window of DM masses between 1 and $100 \mathrm{PeV}$ for such a setup. While conventional searches may lose sensitivity for such a high DM mass, the stochastic gravitational wave background due to the first-order phase transition in this scenario can be detected by planned future facilities. (See Ref. [56] for the projected reach of such facilities.) While this signal depends on the UV parameters that control the thermodynamics of the phase transition, it does not depend on the nature of the portal.

The rest of this paper is organized as follows. In Sec. II, we provide an overview of the cosmology of our dark sector. In Sec. III, we write down and solve the Boltzmann equations that determine the relic abundance of dark matter. In Sec. IV, we provide an overview of the possible phenomenological implications of our dark sector before concluding in Sec. V. We also provide three Appendixes with more details. In Appendix A, we provide more details on the thermodynamics of first-order phase transitions in an expanding universe and detail a simulation we performed to fix the phase transition parameters that enter the relic abundance calculation. In Appendixes B and C, we review some results in the literature for the cross sections and binding energies of heavy quarks and their bound states. For a more condensed discussion than what follows, see the companion paper Ref. [57].

\section{QUALITATIVE OVERVIEW OF COSMOLOGY}

We consider a dark sector with a non-Abelian $S U(3)$ gauge group and a single flavor of heavy quarks $q$ in the fundamental representation

$$
\mathcal{L} \supset-\frac{1}{4} G^{\mu \nu} G_{\mu \nu}+\bar{q}\left(i \gamma_{\mu} D^{\mu}-m_{q}\right) q,
$$

where $G^{\mu \nu}$ is the dark gluon field strength and $m_{q}$ is the dark quark mass with $m_{q} \gg \Lambda$ (in practice, we consider $m_{q} \geq 100 \Lambda$ ), where $\Lambda$ is the dark confinement scale at which a phase transition takes place.

Given that $m_{q} \gg \Lambda$, we expect that such heavy quarks decouple from the thermal bath well before the phase transition, so the phase diagram of this model is very close to that of pure Yang-Mills for $T \ll m_{q}$. Since the heavy quark regime can be well approximated by the pure-gauge regime, the phase transition behavior is almost independent of the number of heavy quark flavors [58]. Then, the only constraining condition on the number of quark flavors is 
asymptotic freedom or, in special cases, asymptotic safety [59]. Various lattice gauge theory studies have established that the $S U(3)$ phase transition takes place at a critical temperature very near the confinement scale, $T_{c} \approx \Lambda$, and is first order [38-41]. We therefore assume that this dark sector features a first-order phase transition exactly at $T_{c}=\Lambda$.

The effects of this phase transition on the relic abundance of DM have been relatively unexplored in previous studies of such a confining gauge sector, e.g., [25]. In this section, we discuss how this phase transition dramatically changes the relic abundance calculation by causing a second stage of significant DM annihilation.

We remain agnostic about how the dark quark mass is generated as it will not affect our study. We also do not commit to any specific portal between the dark sector and the SM. We merely assume such a portal exists and that it keeps the dark sector in kinetic equilibrium with the SM. The portal enables the decay of dark glueballs and mesons to the SM while respecting the dark baryon number symmetry, thus stabilizing the dark baryons. These baryons, which are three-quark bound states, are the DM candidate in this setup. We also assume a symmetric initial condition, $n_{q}=n_{\bar{q}}$.

In this section, we provide an overview of the cosmology of such a sector, focusing primarily on the effect that the phase transition has on the DM relic abundance. The goal is to provide the reader with a broad picture of the various moving parts in this study while leaving some of the more detailed calculations for later sections and Appendix A.

\section{A. Preconfinement epoch}

For high enough temperatures, $T>T_{c}$, the dark sector exists in a deconfined thermal state in which quarks move freely within a gluon bath. Naively, this setup seems at odds with confinement, which requires that colored objects not propagate freely over distances greater than the confinement length $\Lambda^{-1}$. However, qualitatively, these colored quarks can move freely because they are connected to a network of thermal gluons [60]. These gluons screen a quark's color charge so that the quark effectively behaves like a color neutral object, in a process analogous to Debye shielding in plasmas. More quantitatively, lattice simulations have shown that when $T \geq T_{c}$, the potential between two heavy quarks flattens when they are separated by a distance of roughly more than $\Lambda^{-1}$ [38]. In other words, distant quarks in a gluon bath do not influence one another.

In the deconfined phase, the quark relic abundance calculation proceeds analogously to a standard WIMP relic abundance calculation. For large enough dark quark masses, $m_{q} \gtrsim 20 \Lambda$, which will be satisfied for all the parameter space we consider in our analysis, the dominant number changing process $q \bar{q} \leftrightarrow g g$ freezes out before confinement. The Boltzmann equation governing this freeze-out is simply

$$
\dot{n}_{q}+3 H(T) n_{q}=-\langle\sigma v\rangle\left(n_{q}^{2}-\left(n_{q}^{\mathrm{eq}}\right)^{2}\right),
$$

where $H(T)$ is the Hubble constant at temperature $T,\langle\sigma v\rangle$ is the thermally averaged annihilation rate for $q \bar{q} \leftrightarrow g g, n_{q}$ is the quark number density, and $n_{q}^{\mathrm{eq}}$ is its value in thermal equilibrium with a thermal bath of temperature $T$ and with zero chemical potential. Since $T \gg \Lambda$ in this epoch, $\langle\sigma v\rangle$ can be calculated perturbatively [61],

$$
\langle\sigma v\rangle=\zeta \pi \frac{\alpha^{2}\left(m_{q}\right)}{m_{q}^{2}},
$$

where $\alpha\left(m_{q}\right)$ is the dark, strong coupling constant evaluated at the dark quark mass scale and $\zeta$ is a prefactor encapsulating plasma effects and nonrelativistic enhancements (with numerical values presented in Fig. 14). We find that the exact size of this cross section does not qualitatively change the main results of this paper. For further elaboration about this cross section and others, see Appendix B. ${ }^{4}$ For the running coupling constant, we use [62]

$$
\alpha\left(m_{q}\right)=\frac{12 \pi}{\left(11 N_{c}-2 N_{f}\left(m_{q}\right)\right) \log \frac{m_{q}^{2}}{\Lambda^{2}}},
$$

where $N_{f}(\mu)$ is the number of light flavors contributing to the beta function at mass scale $\mu$. We set $N_{c}=3$ and $N_{f}\left(m_{q}\right)=1$.

In the left panel of Fig. 1, we show the resulting quark number density evolution for specific choices of quark mass and confinement scale. A generic obstacle in the study of strong sectors is the uncertainty in determining cross sections. To characterize this uncertainty, we vary the cross section within an order of magnitude around the central value in Eq. (2.3), which produces the green bands.

Importantly, we find that heavy quarks are well separated just before the phase transition begins. To characterize their separation, we define the typical interquark spacing in units of the confinement length,

$$
\xi\left(t_{n}\right) \equiv \frac{\Lambda}{\left(n_{q}\left(t_{n}\right)\right)^{1 / 3}},
$$

where $t_{n}$ is the time at which extensive bubble nucleation starts, i.e., the onset of the phase transition, and $n_{q}\left(t_{n}\right)$ is the number density of the quarks at this time. This quantity measures, in units of $\Lambda^{-1}$, the typical distance between quarks at the onset of the phase transition. When $\xi(t)$ is large, quarks are separated by much more than a

\footnotetext{
${ }^{4}$ In addition to $\bar{q} q$ annihilation, quarks are able to bind into diquarks via the attractive antitriplet channel [61], and diquarks can bind with quarks to form baryons. We have checked that in the pre-confinement epoch and for the parameter space we are considering, this bound state production is negligible (see Sec. III).
} 

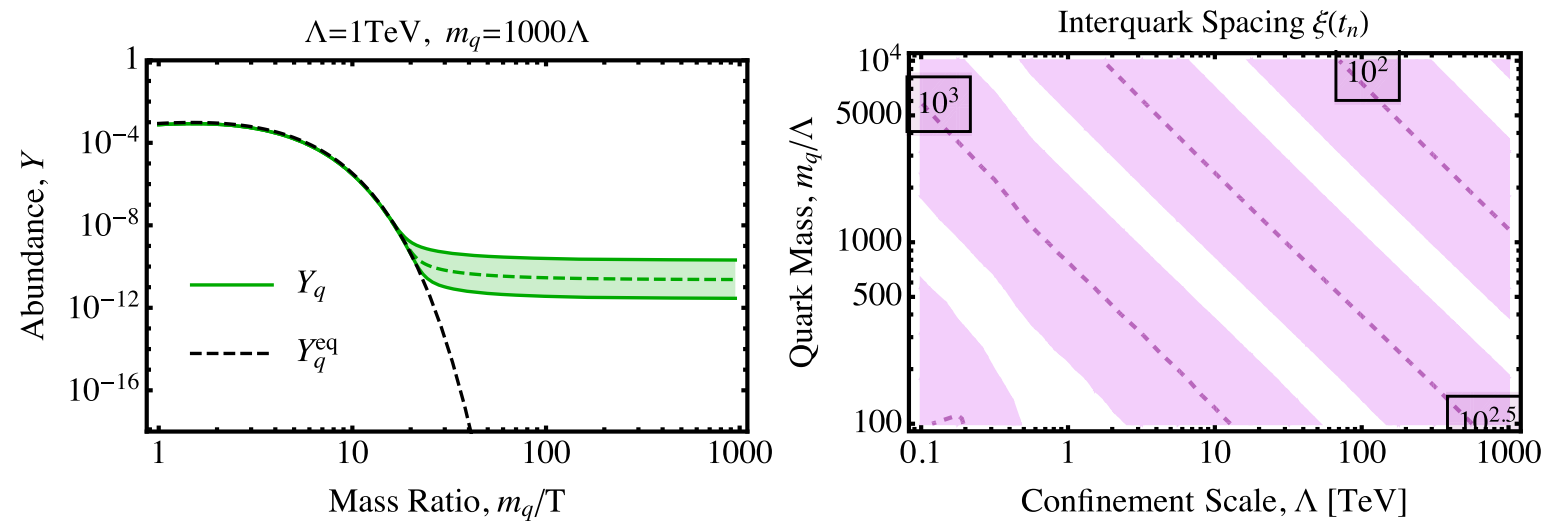

FIG. 1. Left panel: yield (number density normalized by the entropy density of the universe), $Y=\frac{n_{q}}{s}$, of the quarks for a quark mass of $10^{3} \mathrm{TeV}$ and confinement scale of $1 \mathrm{TeV}$. Right panel: average separation of quarks as defined in Eq. (2.5) at the onset of the phase transition for various values of $\Lambda$ and $m_{q} / \Lambda$. In each plot, we vary the cross section by a factor of 10 above or below the central value (dashed lines) in Eq. (2.3) to produce the shaded regions.

confinement length. In the right panel of Fig. 1, we show $\xi\left(t_{n}\right)$ as a function of $m_{q}$ and $\Lambda$. Indeed, quarks are generically further from each other than a confinement length just as the phase transition begins. Were quarks not so well separated, the details of the phase transition would have a less dramatic effect on the DM relic abundance calculation, and we would be able to use the combinatorial method of Ref. [25].

\section{B. Bubble dynamics}

Once the universe cools down to the critical temperature, $T_{c}=\Lambda$, a first-order phase transition begins. Phase conversion cannot occur right at the critical temperature as both phases have the same free energy, so the temperature of the deconfined phase initially cools slightly below $T_{c}$. As the deconfined phase supercools further into a metastable state, bubbles of the confined phase begin to nucleate and expand at a non-negligible rate.

As the deconfined phase is converted to the confined phase, latent heat is released. In contrast to weakly coupled phase transitions, there is no perturbative parameter suppressing the latent heat, meaning that phase conversion will serve as a significant heat source in the temperature evolution of the universe. As a result, the plasma heats back up to a temperature very close to $T_{c}$ quickly after bubble nucleation becomes efficient. Since the nucleation rate is exponentially sensitive to the degree of supercooling, $\left(T-T_{c}\right) / T_{c}$, subsequent nucleation of bubbles is completely suppressed.

For the phase transition to continue, at least some of the bubbles from the brief period of efficient nucleation must continue to grow. To determine the bubble growth rate, we borrow an argument from [52]. As bubbles grow, the local temperature at the bubble walls increases towards $T_{c}$, diminishing the free energy difference between the two phases that drives the expansion. The expansion rate is then limited by the rate at which the wall can cool. The cooling rate is controlled by the temperature gradient between the wall and surrounding fluid-if there were no temperature difference, heat would not flow. Since the wall temperature cannot exceed $T_{c}$ without reversing direction, we assume that this temperature difference is bounded above by the small degree of supercooling $\left(T_{c}-T\right) / T_{c}$. By modeling the heat dynamics near the wall in Appendix A, we estimate that the wall velocity is also bounded above by the degree of supercooling, $v_{w} \leq\left(T_{c}-T\right) / T_{c}$. For simplicity, we assume that $v_{w}$ saturates this bound.

In Fig. 2, we plot the degree of supercooling as a function of time during the bubble expansion stage of the

Temperature Evolution

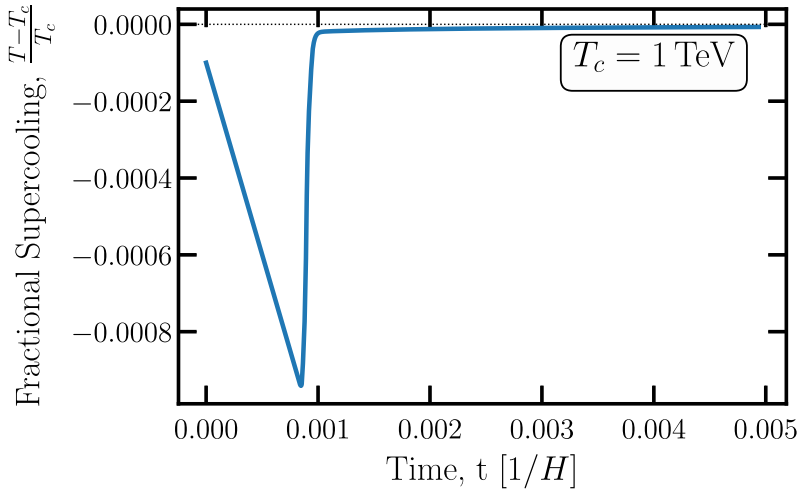

FIG. 2. The degree of supercooling prior to percolation. The temperature supercools until bubbles nucleate efficiently. The nucleated bubbles quickly expand, depositing latent heat that drives $T$ back up to $T_{c}$ and eliminates further bubble nucleation. The temperature then stays roughly constant as latent heat deposited from bubble growth nearly cancels Hubble cooling. This plot ends at percolation, when half of the universe is in each phase. Notice that the timescale of the phase transition is much shorter than the Hubble timescale. 
phase transition. This result comes from a simple simulation that we develop to track the nucleation and growth of bubbles during this epoch. Further details about this simulation can be found in Appendix A. The stages of the phase transition discussed above are visible in this plot. The universe initially supercools through Hubble expansion until bubble nucleation becomes efficient, leading to quick bubble growth and latent heat injection that reheats the universe. The heating and cooling rates then roughly balance one another, leaving the temperature at a value very near $T_{c}$, which suppresses further bubble nucleation as explained above.

Eventually, half of the universe converts to the confined phase. At around this so-called percolation time, most bubbles are in contact with one another and start coalescing. Soon after, we are left with isolated "pockets" of the deconfined phase submerged in a sea of the confined phase. To properly compute the spectrum of shapes and sizes of these pockets would require a full numerical 3D bubble simulation. Instead, to simplify our analysis, we assume that soon after percolation there is a characteristic size of a typical pocket, that pockets can be approximated as spherical, and that the details of the spectrum of pocket shapes and sizes will give only subdominant corrections to our results.

To determine this characteristic pocket size, we first determine the characteristic size of bubbles just before percolation. Using our simulation from Appendix A, we find that at percolation the spectrum of bubble radii peaks strongly at

$$
R_{0} \approx 10^{-6} \times\left(\frac{\Lambda}{M_{p l}}\right)^{-0.9} \frac{1}{\Lambda}
$$

where $M_{p l}=2.4 \times 10^{18} \mathrm{GeV}$ is the reduced Planck mass. We now borrow another argument from [52] that for most values of $\Lambda$, these bubbles coalesce quickly until they reach a larger characteristic size, denoted as $R_{1}$.

The central idea is that small bubbles coalesce and merge quickly into bigger bubbles, and that the timescale for two bubbles in contact to merge becomes longer as bubble sizes grow. Intuitively, the larger the coalescing bubbles, the more matter has to be moved via the bubbles' surface tension, which takes more time. Thus, there is a special bubble size $R_{1}$ above which bubbles merge slower than the timescale over which the phase transition takes place. We find this critical size to be

$$
R_{1} \approx\left(\frac{M_{\mathrm{pl}}}{10^{4} \Lambda}\right)^{2 / 3} \frac{1}{\Lambda}
$$

Figure 3 shows that for $\Lambda \gtrsim 1 \mathrm{TeV}$, the typical size of bubbles just before percolation $\left(R_{0}\right)$ is always smaller than $R_{1}$. Thus, we assume that, for this range of $\Lambda$, at percolation all bubbles quickly coalesce until they reach a size of $R_{1}$. For smaller $\Lambda \mathrm{s}$, we assume that all bubbles will have radius $R_{0}$ instead. We then make the simplifying assumption that the characteristic size of pockets just after percolation is the same as the characteristic size of bubbles just before percolation, i.e.,

$$
R_{i}=\max \left(R_{0}, R_{1}\right)
$$

where $R_{i}$ is the characteristic initial pocket radius after percolation.

It is more complicated to determine the wall velocity of the contracting pocket. The main complication is that quarks are trapped within pockets, which we will show in the next section, and will generically slow down the wall. For now, we assume that we can neglect the effect of the enclosed quarks, which will lead to an overestimate of $v_{w}$.
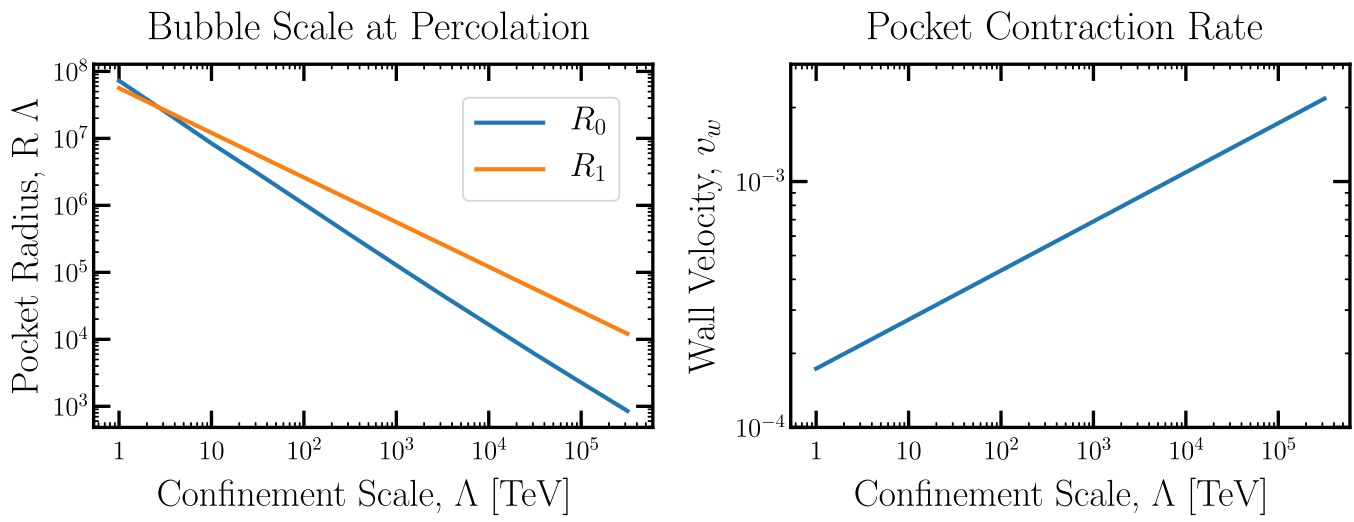

FIG. 3. Left panel: typical radius of bubbles just before percolation (blue line) and the characteristic coalescence radius $R_{1}$ (orange line). For any $\Lambda$ with $R_{0} \leq R_{1}$, we assume that bubbles quickly coalesce and grow to radius $R_{1}$ at percolation. Right panel: asymptotic velocity of the pocket wall during its contraction as a function of the confinement scale when quark pressure is ignored. In the more realistic case where internal quark pressure is allowed to resist the contraction of the pocket, we expect $v_{w}$ to be much smaller and to not necessarily asymptote to a constant value at small radii. 
In Sec. II D, we will revisit the effect these quarks have on $v_{w}$.

In Appendix A, we find that at radii much smaller than $R_{i}$, the pocket contraction rate asymptotes to a constant value, which is shown in the right panel of Fig. 3 and can be fit by

$$
v_{w}(\Lambda) \approx 0.2 \times\left(\frac{\Lambda}{M_{p l}}\right)^{0.2}
$$

In Sec. III, we see that the relic abundance of DM is set while $R \ll R_{i}$, so we can neglect the initial stages when $v_{w}$ varies and treat it as a constant. The pockets' radii therefore shrink as a function of time according to

$$
R\left(t^{\prime}\right)=R_{i}-v_{w} t^{\prime}
$$

where $t^{\prime}$ is the time after percolation.

To the best of our knowledge, the problem of characteristic bubble properties, e.g., the wall velocity and characteristic size at percolation, is not completely settled for first-order phase transitions even in weakly interacting theories (see [63] and the references therein for recent discussions on calculating the wall velocity). In our numerical calculations in Sec. III, we characterize these uncertainties by varying both $v_{w}$ and $R_{i}$ within 1 order of magnitude of the results shown in Fig. 3.

We note here that since the quark temperature is fixed near $T_{c}$ throughout the phase transition, the typical quark velocity is

$$
v_{q} \sim \sqrt{\Lambda / m_{q}}
$$

For the range of parameters that we are interested in, we find $v_{q} \gg v_{w}$. This inequality will become important in the next section when we analyze the effects that the walls have on the quarks.

To summarize, the phase transition begins with an initial, complicated stage of bubble nucleation and growth until bubbles come into contact with one another. It then enters an even more complicated bubble coalescence stage. The space between bubbles is made of pockets of the deconfined phase with the same characteristic size, $R_{i}$. These pockets become isolated and eventually spherical, and then contract initially with a velocity that is determined by the local heat diffusion rate. The contraction rate gradually slows down due to the pressure of the enclosed quarks, and the pockets eventually vanish. The phase transition has been completed at this point, and the universe can proceed with its standard expansion history.

Further details of this phase transition, as well as an overview of the relevant thermodynamics, can be found in Appendix A. Our study of the phase transition's effect on the DM relic abundance is insensitive to many of the details of the phase transition; we merely need an expression for the characteristic initial radius of pockets and their wall velocity, which are respectively provided in Eqs. (2.8) and (2.9). We emphasize that this latter expression for $v_{w}$, which neglects the effect of quark pressure, overestimates the wall velocity during the contraction phase.

\section{Heavy quarks during the phase transition}

During the entire process of bubble nucleation and expansion described in the previous section, bubble walls run into quarks and antiquarks. In this section, we study these encounters in detail and argue that the walls are impermeable to quarks but permeable to color-neutral bound states. While we focus on the interaction between walls and quarks, our conclusions hold for antiquarks as well.

When a wall encounters a quark, the quark can push against it and deform it locally. Whereas in electroweaklike phase transitions a particle is able to penetrate through the wall at the cost of only a finite mass difference [44,45], the energy cost for an isolated quark to enter the confined phase is unbounded [38], preventing it from traveling far into the confined phase. Therefore, a quark can enter a bubble only if it either forms a color-neutral bound state before it enters the bubble or it deforms the wall so that it remains immersed in the color-screening gluon bath (see Fig. 4).

There are two ways in which the quark could form a bound state. First, $\bar{q} q$ pairs could be spontaneously created, binding with the quark as it passes through the wall. We can imagine a scenario as in Fig. 4 in which the quark pushes into the bubble and is connected to a gluon string [60] starting from its initial point of contact with the wall. If the quark were light enough, at some point this stretched string could break into a $\bar{q} q$ pair and the $\bar{q}$ could bind with the incoming quark to form a color-singlet bound state that enters the bubble (see [51] for an example in which this process is efficient). However, for a heavy quark, the string breaking rate is extremely suppressed; this rate can be approximated [64] using the Schwinger mechanism [65],

$$
\left(t_{\text {string }}\right)^{-1} \sim \frac{m_{q}}{4 \pi^{3}} e^{-m_{q}^{2} / \Lambda^{2}} .
$$

The exponential of the square of the large ratio $m_{q} / \Lambda$ makes this string breaking timescale much larger than all other timescales, completely shutting off this process. The inefficiency of string breaking and the quark's inability to pass through bubble walls are the main features distinguishing our model from those that involve light quarks.

The second way a quark could form a bound state is by encountering an antiquark or two quarks somewhere within the deconfined phase, binding, then escaping into the confined phase before the bound state dissociates. These processes are important and will be analyzed in the next section via Boltzmann equations. 


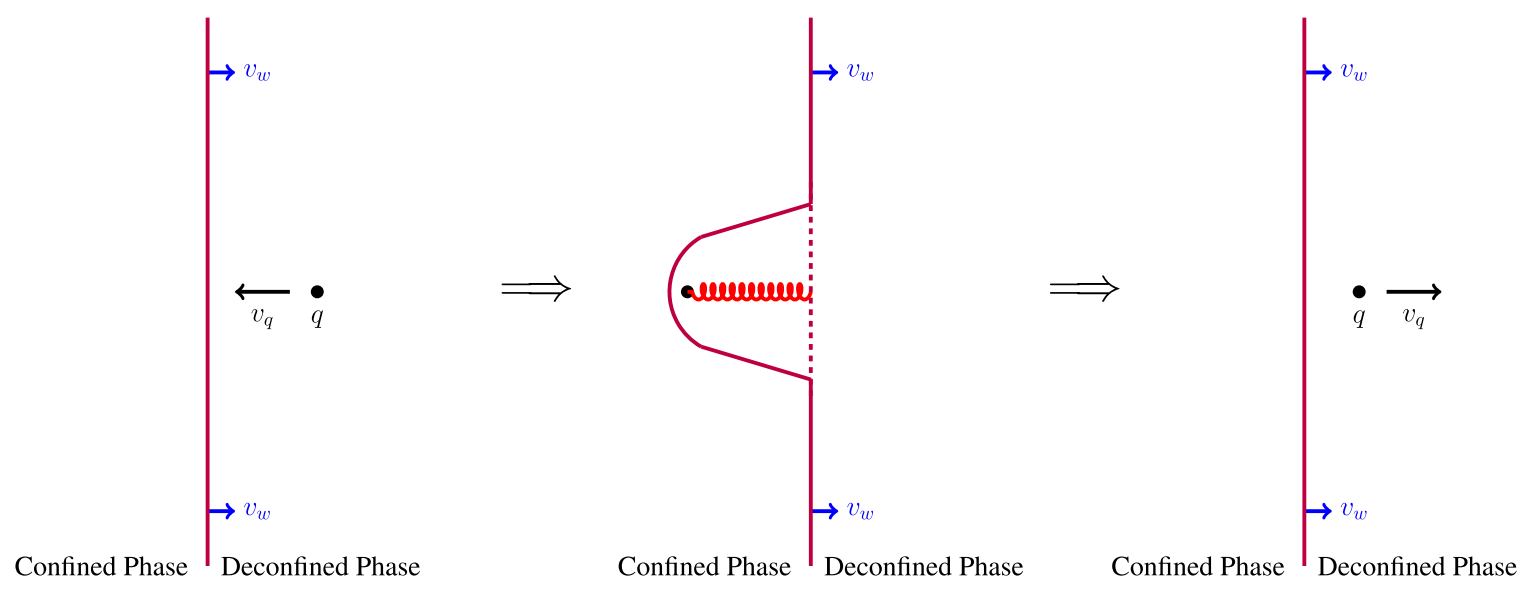

FIG. 4. Depiction of a quark interacting with a phase boundary. As bubbles of the confined phase grow (the wall moves to the right in the figure), their walls run into quarks that move with typical velocity $v_{q}$, which is much larger than the wall velocity $v_{w}$ (left configuration). The quark locally deforms the wall (center configuration), introducing an opposing force via surface tension. One can think of the quark as connected to the deconfined phase through a gluon string [60]. Since string breaking is shut off, i.e., the quarkgluon configuration does not have sufficient energy to pull a heavy $\bar{q} q$ pair out of the thermal background, eventually the quark comes to a halt and then rebounds back into the deconfined phase with its initial speed (right configuration).

If a quark has not managed to bind into a color singlet state by the time it reaches a bubble wall, it deforms the wall to avoid entering the confined phase. As the wall deforms, its surface area increases, which increases the energy of the system. The surface tension therefore creates a force that opposes this deformation. If we estimate this force to be of order $\Lambda^{2}$ on dimensional grounds, then we find that the timescale for the surface tension to restore the shape of the wall and reverse the quark's velocity is

$$
t_{\text {rebound }} \sim \frac{v_{q}}{\dot{v}_{q}} \sim \frac{v_{q}}{\Lambda^{2} / m_{q}}=\sqrt{\frac{m_{q}}{\Lambda}} \frac{1}{\Lambda} .
$$

This rebound timescale is much shorter than the string breaking timescale. It is also orders of magnitude smaller than the phase transition timescale, which we find in Appendix $\mathrm{A}$ to be $t_{\mathrm{PT}} \sim 10^{-2} H^{-1} \sim 10^{-2} M_{\mathrm{pl}} / \Lambda^{2}$ (also, see Fig. 2). Finally, the pocket contraction timescale is of order $t_{\text {contract }} \sim R / v_{w}$. Since $R>\Lambda^{-1}$ and we find that $v_{w} \lesssim 10^{-3}$, we have $t_{\text {contract }}>10^{3} / \Lambda$. Since we only consider quark masses that satisfy $\sqrt{m_{q} / \Lambda} \leq 10^{2}$ in this paper, we have $t_{\text {rebound }} \ll t_{\text {contract }}$. Since this rebound timescale is the shortest timescale in the problem, quarks rebound off walls very quickly before any other process can take place. Therefore, the bubble walls act like very stiff surfaces that quickly reflect quarks that come into contact with them.

As these bubbles grow, the walls sweep quarks and antiquarks into the ever-shrinking deconfined regions, increasing the quark density over time. Moreover, since $v_{q} \gg v_{w}$, quarks that are swept in can quickly travel through the shrinking deconfined region and maintain homogeneity, meaning that $n_{q}$ is independent of position in the pocket throughout the phase transition. Eventually, these particles end up inside the isolated pockets formed toward the final stage of the phase transition (see Fig. 5).

We note that in models with additional light (though not massless) quarks, there would be no first-order phase transition [66], and even if such a transition did exist, quarks would easily pass through bubble walls and would most likely be unaffected by the phase transition.

Within any fixed volume of the universe, including the isolated pockets, the baryon number is a fluctuating random variable. Although the baryon number averaged over all pockets must be zero due to our symmetric initial condition, any given pocket is expected to have an overabundance or underabundance of quarks relative to antiquarks, which we call the pocket asymmetry, $\eta$. We find that the initial total number of quarks in a pocket, $N_{q}^{\text {initial }}$, is large, so by the central limit theorem, the standard deviation of fluctuations above and below the mean is $\sqrt{N_{q}^{\text {initial }}}$. Therefore, no matter how efficient $\bar{q} q$ annihilation processes are in these contracting pockets, on average, at least a fraction

$\eta_{\mathrm{rms}} \equiv \sqrt{\left\langle\eta^{2}\right\rangle}=\sqrt{N_{q}^{\text {initial }}} / N_{q}^{\text {initial }}=1 / \sqrt{N_{q}^{\text {initial }}}$

of the initial quarks (or antiquarks) in a pocket will survive. This observation will have important consequences for our relic abundance calculation in the next section.

Once isolated pockets have formed and their asymmetries have been set, they will contract and compress quarks and antiquarks until formerly frozen-out interactions turn back on. These interactions include $\bar{q} q$ annihilation as well as $q+q$ binding via the attractive antitriplet channel [61]. 

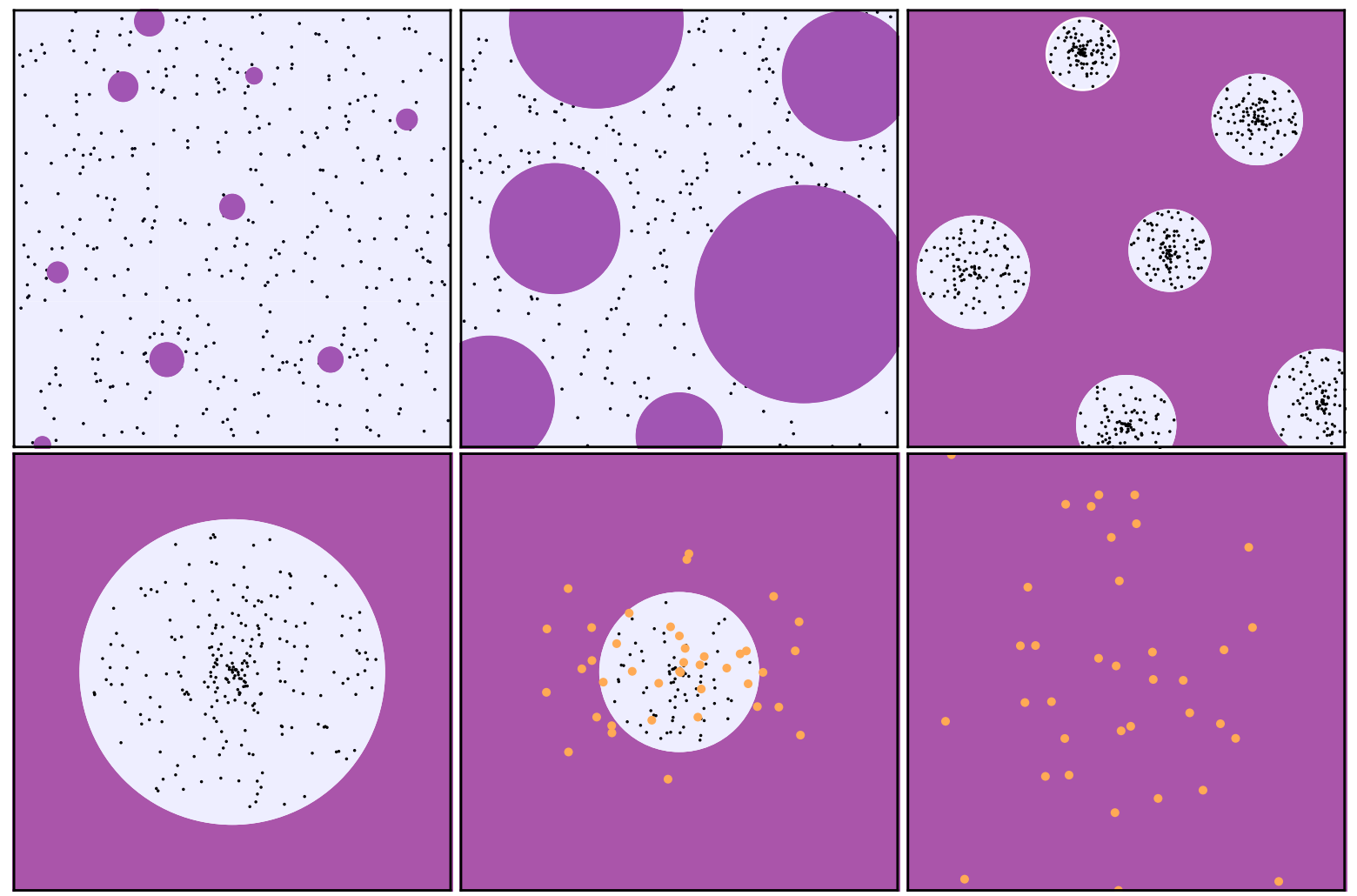

FIG. 5. Schematic illustration of different stages of the phase transition and its effect on the DM abundance. Violet indicates the confined phase and light blue the deconfined phase. Top-left panel: Once the temperature drops slightly below $T_{c}=\Lambda$, bubbles of the confined phase begin nucleating everywhere. The nucleated bubbles start growing and push quarks (black dots) around. Top-middle panel: The bubbles grow to a point where a $\mathcal{O}(1)$ fraction of the universe has converted into the confined phase. At this point, bubbles start coalescing and quickly grow larger. Top-right panel: As the bubbles keep growing and combining, eventually we are left with isolated pockets of the deconfined phase submerged in a sea of the confined phase. Bottom-left panel: A single isolated pocket with quarks trapped inside it. Each pocket contracts as the phase transition continues. Bottom-middle panel: The particles in the pocket are compressed, and their interactions recouple. During this phase, the particles can either annihilate or bind with one another. Nonsinglet states cannot enter the confined phase, but once they form color-neutral bound states (orange dots), they can escape into the confined phase. Bottom-right panel: In the end, the pockets vanish, and only the fraction of quarks that ended up inside color-neutral baryons survive. These particles diffuse away from the original pocket's position due to their local overdensity.

As these diquarks build up their occupation number, they can eventually bind with quarks to form color-singlet baryons that can quickly fly out of the pocket. ${ }^{5}$ These escaping stable baryons constitute the DM candidate of our model, while the rest of the particles eventually dump their energy into the SM sector through an unspecified portal interaction.

We define a survival factor as the fraction of quarks and antiquarks that escape the pocket within baryons and antibaryons,

$$
\mathcal{S} \equiv \frac{N_{q}^{\text {survived }}}{N_{q}^{\text {initial }}}
$$

In the next section, we write down the Boltzmann equations governing the quark dynamics within contracting pockets

\footnotetext{
${ }^{5}$ Notice that the formation of baryons through an intermediate diquark is more efficient than the formation of baryons via direct 3-body recombination, which we ignore.
}

and calculate this survival factor. As remarked above, $\mathcal{S}$ is bounded below by the asymmetry of a given pocket, and the expectation value of this lower bound is

$$
\mathcal{S} \geq \eta_{\mathrm{rms}}=\frac{1}{\sqrt{N_{q}^{\text {initial }}}} .
$$

After these surviving baryons escape the pockets and after the phase transition is eventually completed, these baryons continue to diffuse away until they reestablish homogeneity in the universe. If the asymmetry bound is not saturated, baryons and antibaryons can continue to annihilate as they diffuse outside of the pocket. A more detailed study of this final annihilation stage requires integrating inhomogeneous Boltzmann equations, which we leave for future work.

In summary, dark matter undergoes a short squeeze, where collapsing bubbles during the phase transition induce a second stage of rapid annihilation that drastically 
depletes the universe's preexisting stock of dark matter. This extra annihilation after freeze-out opens up parameter space that had previously been ruled out due to overproduction of DM. We will show that this effect allows for thermal DM to be heavier than the conventional unitarity bound of $\sim 300 \mathrm{TeV}$ [53].

\section{Quark pressure on the wall}

Although we have considered the effect that the wall has on the trapped quarks, we have ignored the effect that the trapped quarks have on the wall. In this section, we argue that the trapped quarks generically slow down the contraction rate of the wall. ${ }^{6}$

Much like a piston, a pocket wall can contract only if it works on the enclosed gas of heavy quarks. Since we have assumed that this gas is thermally coupled to the rest of the SM bath, which has a much larger heat capacity than that of the dark sector, the quarks contract at constant temperature. Using an ideal gas equation of state, we can write the pressure of this quark gas as $p_{q}=n_{q} T$. The work that the wall does on the gas when it contracts by an amount $d R$ is therefore $p_{q} d V=4 \pi R^{2} n_{q} T d R$.

The forces that are responsible for this change of pocket radius are the surface tension and net gluonic pressure, the latter of which is directed inward whenever $T<T_{c}$. We will show in the next section that during the earliest stages of pocket contraction, quark interactions are inefficient, and the total number of quarks in the pocket is initially conserved. As a result, when the pocket shrinks, the work required to contract the pocket grows like $R^{-1}$. At the same time, the work that the surface tension and net gluonic pressure do when contracting the pocket by $d R$ is proportional to the change in area and volume, respectively, so they shrink like $R$ and $R^{2}$. Altogether, as $R$ contracts, the forces pushing out grow while the forces pushing in shrink. We therefore expect that $v_{w}$ decreases with decreasing $R$.

As this physics involves nonequilibrium, strong dynamics, we cannot reliably compute $v_{w}$ as a function of $R$. Instead, in the remainder of this section, we will argue that the effect of quark pressure is to slow down $v_{w}$ by orders of magnitude relative to the upper bound of Eq. (2.9) we computed when we neglected the quark pressure. For more details relevant to the following discussion, we refer the reader to Appendix A 4.

When we simulate pocket contraction while keeping track of the quark density within a pocket (see next section), we find that there always comes a point when the quark pressure has grown to such an extent that, were we to suddenly include it, the quark pressure would exactly oppose all inward-pointing forces. This point of mechanical equilibrium is defined by $\sigma d A+\left(\sum p\right) d V=0$, where $\sigma$ is the surface tension, $d A$ the change in surface area, $d V$ the

\footnotetext{
${ }^{6}$ We thank Filippo Sala for pointing out this effect.
}

change in volume, and $\sum p$ the sum of pressures acting on the wall. (Inward-pointing pressures are defined to be positive while outward facing pressures are negative.) If we were to include the effects of quark pressure, the motion of the wall would suddenly become calculable since the state of the wall would be determined by equilibrium physics. The pocket would slow down and proceed to adiabatically shrink while maintaining mechanical equilibrium. Number changing processes would deplete $n_{q}$, diminishing the quarks' outward-pointing pressure, and the universe would supercool further, increasing the net gluonic inward-pointing pressure. We find that in this scenario, $v_{w}$ suddenly drops by orders of magnitude when mechanical equilibrium is achieved, and $v_{w}$ steadily decreases by many more orders of magnitude as the pocket contracts.

The discontinuous drop in $v_{w}$ signals a breakdown of our assumption that quark pressure was negligible before mechanical equilibrium was achieved. This simulation merely demonstrates that it is inconsistent to neglect quark pressure and that it can potentially slow down the pocket contraction rate by orders of magnitude. We therefore expect that a more realistic simulation that correctly includes the effects of quark pressure from the very beginning will lead to a $v_{w}$ that gradually decreases from our upper bound of Eq. (2.9), which eventually overestimates $v_{w}$ by orders of magnitude.

We will use the results of our pocket evolution simulations to calculate a few parameters that enter the Boltzmann equations that govern the abundances of various bound states in the pocket. While the expression for the pocket radius, Eq. (2.8), is robust to the uncertainties introduced by quark pressure, we argue that the wall velocity $v_{w}$ is sensitive to this uncertainty. In the next section, we will study the evolution of the bound state abundances in the pocket in two extreme cases: (i) when the effect of quark pressure on $v_{w}$ is completely ignored, or (ii) when its effect dramatically reduces $v_{w}$.

\section{BOLTZMANN EQUATIONS DURING COMPRESSION}

As described above, toward the end of the phase transition, the deconfined regions form isolated pockets that contain all of the dark quarks. In this section, we describe the dynamics of the dark quarks and their bound states as the contracting pockets compress them. The Boltzmann equations that we solve keep track of the many processes by which quarks either ultimately annihilate into gluons or form baryons that escape the pockets and become dark matter. We solve the Boltzmann equations for a typical pocket with initial characteristic radius $R_{i}$ and pocket asymmetry set to its root-mean-square value, $\sqrt{N_{q}^{\text {initial }}}$. We assume that the $\mathcal{S}$ of this typical pocket is approximately equal to $\mathcal{S}$ averaged over the full distribution of 
initial pocket radii and pocket asymmetries. The total number of DM particles that survive until today will then equal the total number of DM particles entering the phase transition times $\mathcal{S}$.

\section{A. Ingredients of the Boltzmann equations}

We begin by listing the degrees of freedom that we will include in our Boltzmann equations, which have been tabulated in Table I. We have neglected a host of exotic hadronic bound states like tetraquark and pentaquark states because we assume that they are unstable and promptly decay to the states listed in Table I. We also do not consider excited states of any of the bound states. To simplify the notation, we label states by their quark number throughout the text (for example, a baryon state is a 3 state while an antidiquark is a -2 state).

We also neglect the mesons $\bar{q} q$ in our analysis. This can be justified by comparing their decay rate to the fastest annihilation rate that we will encounter (see Appendix B),

$$
\begin{aligned}
\Gamma_{\bar{q} q} & \sim \alpha^{5} m_{q}, \\
\langle\sigma v\rangle_{\max } n_{q} & \sim \frac{1}{\alpha^{3}} \frac{\alpha^{2}}{m_{q}^{2}}\left(\frac{\Lambda}{\xi(t)}\right)^{3}, \\
\Rightarrow \frac{\langle\sigma v\rangle_{\max } n_{q}}{\Gamma_{\bar{q} q}} & \sim\left(\frac{1}{\xi(t) \alpha^{2}} \frac{\Lambda}{m_{q}}\right)^{3} \ll 1,
\end{aligned}
$$

where the last inequality is obtained because we have heavy quarks $\left(m_{q} / \Lambda \geq 100\right)$ and the interquark spacing in units of $\Lambda$ satisfies $\xi(t) \geq 1$. Such a fast meson decay rate ensures that these states are kept in equilibrium so that their number density is negligibly small. We have also verified numerically that including the mesons in our Boltzmann equations below has a negligible effect on our results.

Let us now look into the Boltzmann equation for the particles in Table I as they are compressed by the contracting pockets. We start with the Liouville operators. For the colored particles, i.e., \pm 1 (quarks/antiquarks) and \pm 2 (diquarks/antidiquarks), we have

$$
L[i]=\dot{n}_{i}-3 \frac{v_{w}}{R} n_{i}, \quad i=1,2,
$$

TABLE I. Different degrees of freedom entering the Boltzmann equations of the contracting pockets. We use the quark number of each state to refer to them throughout the text. The existence of antiparticles, with negative dark quark numbers and conjugate representations under $S U(3)$, is implied.

\begin{tabular}{lcc}
\hline \hline State & Dark quark number & Color representation \\
\hline Gluons & 0 & $\mathbf{8}$ \\
Quark & 1 & $\mathbf{3}$ \\
Diquark & 2 & $\overline{\mathbf{3}}$ \\
Baryon & 3 & $\mathbf{1}$ \\
\hline \hline
\end{tabular}

where the second term captures the effect of pocket compression. Notice that we have not included the usual factor of $+3 H n_{i}$ for the dilution of space due to Hubble expansion. As argued above, $t_{\mathrm{PT}} \ll H^{-1}$. Therefore, the Hubble dilution rate is negligible during the phase transition and can be ignored.

For the color-neutral particles, i.e., baryons and antibaryons, the compression term will be absent. Unlike the colored particles, the baryons are not constrained by confinement to remain in the deconfined pockets. The baryons formed in the pocket can then be thought of as a gas created in a container without walls. The gas of baryons will thus escape with a rate governed by its internal pressure or, equivalently, by the thermal velocities of the baryons. ${ }^{7}$

Once the baryons escape the pocket, they are no longer tracked by the Boltzmann equations. So we must include baryon escape as a sink term in our Boltzmann equations, which we do by modifying the Liouville operator,

$$
L[3]=\dot{n}_{3}+3 \frac{v_{q}}{R} n_{3} .
$$

To derive this escape rate, consider a small time step $d t$. In each time step, the pocket radius contracts by $v_{w} d t$. The typical baryon moves a distance of about $v_{q} d t$, where we ignore the distinction between the baryon and quark velocities. We then overestimate the escape rate by an $\mathcal{O}(1)$ factor by assuming that all baryons at the edge of the bubble move radially outward, giving a total number of escaped baryons of

$$
d N_{3}^{\mathrm{esc}}=4 \pi R^{2} n_{3}(R)\left(v_{q}+v_{w}\right) d t .
$$

Combining this with the rate of change for pocket volume gives the density loss rate due to baryon escape used in Eq. (3.3).

It will be convenient to track the evolution of the total number of particles in a pocket as opposed to number densities. Define the pocket volume,

$$
V(R)=\frac{4 \pi}{3} R^{3} .
$$

Multiplying the number density of species $i$ by the volume of a pocket then gives the total number of species $i$ in the pocket,

$$
N_{i} \equiv V n_{i}
$$

\footnotetext{
${ }^{7}$ Notice that the justification for why baryons in the pocket are homogeneously distributed is different than that of the quarks and diquarks. Gradients in the baryon density naturally arise as the baryons flow from their high density points of creation to the low density exterior of the pockets. However, a homogeneous component of baryons is constantly being produced within a pocket due to the binding of (homogeneously distributed) quarks and diquarks. We find that the rate of production is faster than the escape rate, so the baryon density in the pocket remains homogeneous to a good approximation.
} 
It will also be convenient to replace the time coordinate with $R$ using Eq. (2.10). We can then rewrite the Liouville operators as

$$
\begin{gathered}
L[i]=-\frac{v_{w}}{V} N_{i}^{\prime}, \quad i=1,2, \\
L[3]=-\frac{v_{w}}{V}\left(N_{3}^{\prime}-\frac{3}{R} \frac{v_{q}+v_{w}}{v_{w}} N_{3}\right),
\end{gathered}
$$

where $N^{\prime} \equiv d N / d R$ and we have used $\dot{R}=-v_{w}$.

Now that we have dealt with the Liouville operators, we write down the collision operators. We are only concerned with 2-to- 2 processes since $n$-to- 2 processes are Boltzmann suppressed while 2-to- $n$ processes are suppressed by extra factors of $\alpha\left(m_{q}\right)$ and phase space factors. We denote each of these terms by the following notation,

$$
\begin{aligned}
\langle(a, b) \rightarrow(\alpha, \beta)\rangle & =\langle\sigma v\rangle_{a b \rightarrow \alpha \beta}\left(n_{a} n_{b}-n_{\alpha} n_{\beta} \frac{n_{a}^{e q} n_{b}^{e q}}{n_{\alpha}^{e q} n_{\beta}^{e q}}\right) \\
& =\frac{\langle\sigma v\rangle_{a b \rightarrow \alpha \beta}}{V^{2}}\left(N_{a} N_{b}-N_{\alpha} N_{\beta} f_{a b, \alpha \beta}\right),
\end{aligned}
$$

with $a, b, \alpha, \beta=0, \pm 1, \pm 2, \pm 3$, and $f_{a b, \alpha \beta} \equiv \frac{N_{a}^{e q} N_{b}^{e q}}{N_{\alpha}^{e q} N_{\beta}^{e q}}$. For gluons we have $n_{0}=n_{0}^{(e q)}$; i.e., the gluons are always in equilibrium.

Once we have identified all the important interactions to be included in our Boltzmann equations, we can write down the complete system of differential equations for $N_{i}(R)$. We supply these equations with the initial conditions, which were derived in Sec. II. The initial pocket radius is $R_{i}$ while the initial quark number in the pocket, $N_{1}$, is found by multiplying the number density result of the preconfinement freeze-out calculation in Eq. (2.2) by $\frac{4 \pi}{3} R_{i}^{3}$. We find that the initial conditions for $N_{2}$ and $N_{3}$ are irrelevant, as they quickly approach an equilibrium value independent of whatever values we initially choose (so long as $N_{2}, N_{3} \ll N_{1}$ initially). All that is left is to write down these equations and solve them.

\section{B. Complete set of Boltzmann equations}

The complete set of Boltzmann equations is

$$
\begin{aligned}
L[i]= & C[i], \quad i=1,2,3 . \\
C[1]= & -\langle(-3,1) \rightarrow(-1,-1)\rangle-\langle(-3,1) \rightarrow(-2,0)\rangle+2\langle(3,-1) \rightarrow(1,1)\rangle \\
& +\langle(3,-2) \rightarrow(1,0)\rangle-\langle(1,-1) \rightarrow(0,0)\rangle+\langle(2,2) \rightarrow(3,1)\rangle-2\langle(1,1) \rightarrow(2,0)\rangle \\
& +\langle(-3,2) \rightarrow(-2,1)\rangle+\langle(2,-2) \rightarrow(1,-1)\rangle+\langle(2,-1) \rightarrow(1,0)\rangle \\
& -\langle(2,1) \rightarrow(3,0)\rangle-\langle(-2,1) \rightarrow(-1,0)\rangle+\langle(3,-3) \rightarrow(1,-1)\rangle, \\
C[2]= & \langle(1,1) \rightarrow(2,0)\rangle-\langle(-3,2) \rightarrow(-1,0)\rangle+\langle(3,-1) \rightarrow(2,0)\rangle \\
& -\langle(2,-2) \rightarrow(0,0)\rangle+\langle(3,-2) \rightarrow(2,-1)\rangle+\langle(3,-3) \rightarrow(2,-2)\rangle \\
& -\langle(2,-1) \rightarrow(1,0)\rangle-2\langle(2,2) \rightarrow(3,1)\rangle-\langle(2,1) \rightarrow(3,0)\rangle \\
& -\langle(-3,2) \rightarrow(-2,1)\rangle-\langle(2,-2) \rightarrow(1,-1)\rangle, \\
C[3]= & \langle(2,1) \rightarrow(3,0)\rangle+\langle(2,2) \rightarrow(3,1)\rangle-\langle(3,-3) \rightarrow(0,0)\rangle-\langle(3,-1) \rightarrow(2,0)\rangle \\
& -\langle(3,-1) \rightarrow(1,1)\rangle-\langle(3,-3) \rightarrow(1,-1)\rangle-\langle(3,-3) \rightarrow(2,-2)\rangle \\
& -\langle(3,-2) \rightarrow(2,-1)\rangle-\langle(3,-2) \rightarrow(1,0)\rangle
\end{aligned}
$$

where $\langle(\cdot, \cdot) \rightarrow(\cdot, \cdot)\rangle$ is defined in Eq. (3.9). The right-hand side consists of all interactions that are consistent with quark number conservation. We also make the approximation that

$$
N_{i}=N_{-i}
$$

While this equality is not strictly satisfied due to the pocket asymmetry, we are able to satisfy it because only one of three scenarios can occur: Either (i) the symmetric component is never depleted to the point that the asymmetry is important, (ii) it is completely depleted and the accidental asymmetric abundance is all that survives, or (iii) the symmetric and the asymmetric components are comparable and our answer is off by an $\mathcal{O}(1)$ factor. As argued before, this asymmetry introduces a lower bound on $\mathcal{S}$ [Eq. (2.16)].

Despite Eq. (3.10) having numerous terms, solving these equations numerically is rather straightforward. For convenience, we list the important parameters entering into these equations and their reference values in Table II. We remind the reader that Eq. (2.9) overestimates $v_{w}$ since it neglects the quark pressure's ability to oppose pocket 
TABLE II. Relevant quantities in the Boltzmann equations and our expression for each. More discussion on how we treat $v_{w}$ is included in the main text.

\begin{tabular}{lcccccc}
\hline \hline Quantity & $v_{w}$ & $R_{i}(\Lambda)$ & $\xi\left(t_{n}\right)$ & $v_{q}$ & $\sigma v$ & Binding energies \\
\hline Central value & See main text & Eq. (2.8) & Eq. (2.5) & Eq. (2.11) & See Appendix B & See Appendix C \\
\hline \hline
\end{tabular}

contraction. As we will discuss below, we can bracket the effect that a slower $v_{w}$ would have on the final DM relic abundance quite robustly; see Sec. III E for further details. We also reemphasize that we have used simple approximations for some of the other quantitiesparticularly the bubble radius - and a rigorous determination of them is only possible through more extensive numerical calculations.

In Fig. 6, we show the solution of the Boltzmann equations in Eq. (3.10) for a specific quark mass and confinement scale when we neglect quark pressure and use Eq. (2.9) for the pocket wall velocity. There are a number of important observations to be made about this figure. First, the fractions of diquarks and baryons are initially very small, justifying why we did not include them in our calculations prior to pocket formation. Next, as the pockets contract, the number of bound states initially grows while the number of free quarks decreases due to binding or annihilation to gluons. As the number of free quarks decreases, the annihilation or escape of bound states becomes more important than their production, so their occupation numbers reach a maximum and monotonically decrease from there. Finally, we see that each step in the chain of bound state formation $(q+q+q \rightarrow q q+q \rightarrow q q q)$ results in a suppression; i.e., the total number of diquarks is suppressed compared to the total number of free quarks, while the total number of baryons is suppressed compared to the diquarks. We anticipate that had we started with a larger $S U(N)$ gauge group $(N \geq 4)$, the bound states with higher quark numbers would have been further suppressed and the final DM survival factor would be lower. We leave a more detailed analysis of this scenario to future work.

Finally, to calculate the survival factor, we simply integrate Eq. (3.4) to calculate the total number of baryons that escaped during the contraction of the pocket and normalize to the initial quark number in the pocket. Rewriting Eq. (3.4) in terms of $N$ and $R$, we find

$$
\mathcal{S}_{\text {symm }}=\frac{3 \int d N_{3}^{\mathrm{esc}}}{N_{q}^{\mathrm{initial}}}=\frac{9}{N_{1}\left(R_{i}\right)} \int d R \frac{v_{q}+v_{w}}{v_{w} R} N_{3}(R),
$$

where we have used Eqs. (2.10) and (3.6) to change variables, and the subscript in $\mathcal{S}_{\text {symm }}$ is to indicate that this is the survival factor of the symmetric component of the dark quarks. The factor of 3 in the first equality accounts for
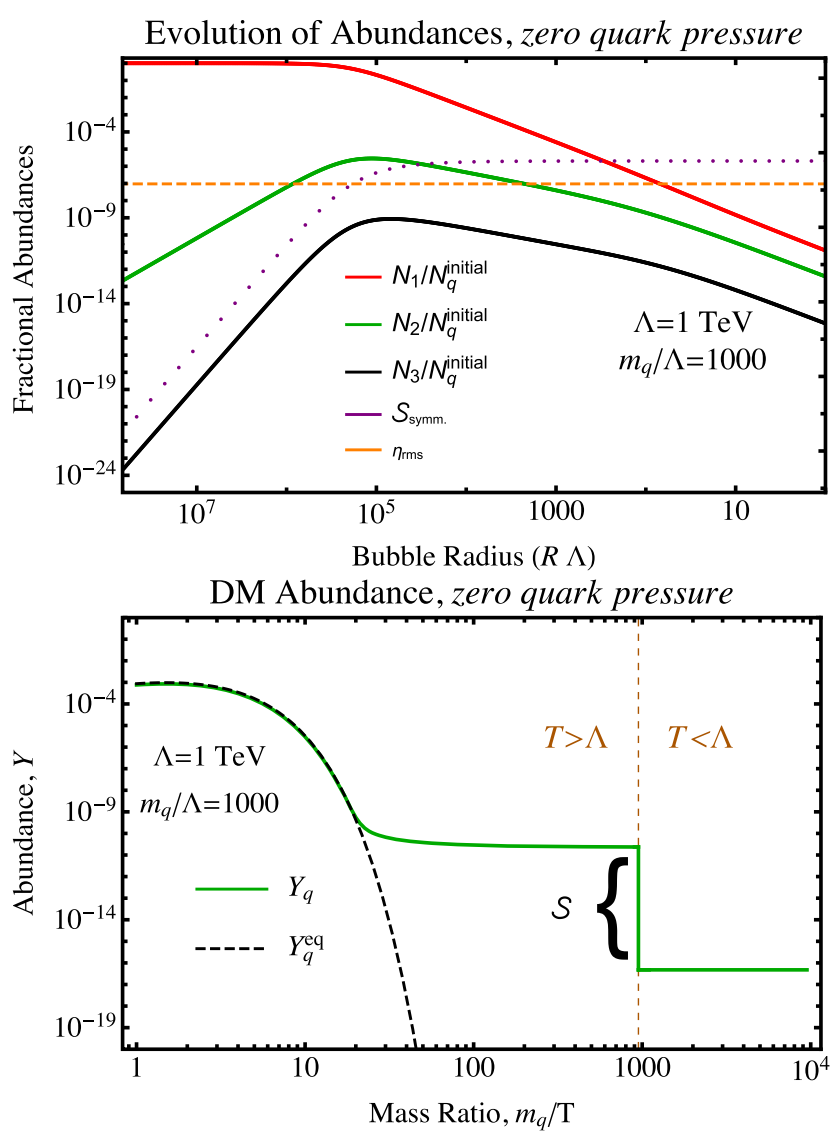

FIG. 6. Top panel: evolution of the fractional number of free quarks (red), diquarks (green), and baryons (black) inside the pocket, normalized to the initial quark number. We neglect the effect of quark pressure on $v_{w}$ in solving the Boltzmann equations for this plot, meaning that we use Eq. (2.9) for $v_{w}$. As the phase transition proceeds, the pocket radius decreases. Initially, almost all the quarks are unbound. As the pocket contracts, more bound states are formed and fewer quarks are found as free particles. As their numbers increase, the various states' annihilation rates increase as well. At some point, their production and annihilation rates are comparable, and the number of bound states inside the pocket reaches its maximum. The accumulative surviving fraction assuming zero pocket asymmetry predicted by Eq. (3.12) is denoted by the dotted purple line. The asymptotic value of this line is equal to $\mathcal{S}_{\text {symm. }}$. We denote the asymmetric lower bound on $\mathcal{S}$ from Eq. (2.16) by the orange dashed line. Bottom panel: DM abundance evolution for this mass and confinement scale. The $T>\Lambda$ region is similar to Fig. 1 ; the confinement takes place at $T=\Lambda$ and gives rise to the abundance suppression predicted by Eq. (3.13). 
the fact that three quarks exists within every baryon that escapes.

Note that in deriving this result we assumed no asymmetry exists in the pocket. Combining this result with the lower bound on $\mathcal{S}$ from the asymmetry component, Eq. (2.16), we have

$$
\mathcal{S}=\max \left\{\mathcal{S}_{\text {symm. }}, \eta_{\text {rms }}\right\} .
$$

In Fig. 6, we show $\mathcal{S}_{\text {symm }}$ and $\eta_{\text {rms }}$ as well. We find that for the chosen $\Lambda$ and $m_{q}$, while neglecting the effect of quark pressure on the wall velocity, $\mathcal{S}_{\text {symm }} \geq \eta_{\mathrm{rms}}$; i.e., the local pocket asymmetry is not saturated during the contraction, but $\mathcal{S}$ is within $\sim 1$ order of magnitude of this asymmetry bound. In fact, we find that this is true for all the points in the parameter space that we study. In the upcoming section we will describe how we can leverage this observation to bracket the range of parameter space that gives rise to the correct DM relic abundance.

\section{Analytic approximation}

While the Boltzmann equations in Eq. (3.10) can be solved numerically, the large number of terms involved can muddle one's intuition. In this section, we develop a simple analytic approximation for solving these equations and determining $\mathcal{S}_{\text {symm }}$.

From the full set of interactions included in Eq. (3.10), we identify and neglect all but the most relevant processes that provide a closed set of equations with an analytic, asymptotic solution that shows good qualitative agreement with the numerical treatment. The subset of processes that we include are the formation of diquarks and the subsequent capture of quarks that lead to the formation of baryons. The reduced set of Boltzmann equations is then

$$
\begin{aligned}
-\frac{v_{w}}{V} N_{1}^{\prime}= & -\langle(1,-1) \rightarrow(0,0)\rangle-2\langle(1,1) \rightarrow(2,0)\rangle-\langle(2,1) \rightarrow(3,0)\rangle+\langle(2,-1) \rightarrow(1,0)\rangle, \\
& -\frac{v_{w}}{V} N_{2}^{\prime}=-\langle(2,-2) \rightarrow(0,0)\rangle-\langle(2,1) \rightarrow(3,0)\rangle+2\langle(1,1) \rightarrow(2,0)\rangle, \\
& -\frac{v_{w}}{V} N_{3}^{\prime}=-\langle(3,-3) \rightarrow(0,0)\rangle-\langle(3,-1) \rightarrow(2,0)\rangle+\langle(2,1) \rightarrow(3,0)\rangle-\frac{d N_{3}^{\mathrm{esc}}}{d R} .
\end{aligned}
$$

The analytic solution for this set of equations is obtained by relying on several assumptions.

(i) The initial dark quark abundance $N_{q}^{\text {initial }}$ is determined by the preconfinement freeze-out of the elementary constituents.

(ii) As long as the annihilation rate and the baryon escape rate in the contracting pocket is slower than the pocket contraction rate $v_{w} / R$, the total quark number is conserved. Once those rates are of the same order, the annihilation process "recouples," and the free quark abundance drops toward zero. The condition $\Gamma_{\text {ann }} \approx N_{q}^{\text {initial }}\langle\sigma v\rangle_{1(-1) \rightarrow 00} / V=v_{w} / R$ defines the recoupling pocket radius

$$
R_{\mathrm{rec}}=\sqrt{\frac{3 N_{q}^{\text {initial }}\langle\sigma v\rangle_{1(-1) \rightarrow 00}}{\left(4 \pi v_{w}\right)}} .
$$

(iii) The initial number of bound states, $N_{X}(X=2,3)$, is negligible. As the pocket contracts, bound states start forming. Thus, we can write $N_{X} \sim R^{-n}$, with $n>0$, which implies $N_{X}^{\prime} \sim N_{X} / R$. Inserting this into the Boltzmann equation shows that there is a small parameter controlling the rate of change, which is proportional to $\delta=N_{X}\langle\sigma v\rangle /\left(R^{2} v_{w}\right) \propto$ $\langle\sigma v\rangle / R_{1}^{2} \sim\langle\sigma v\rangle \Lambda^{10 / 3} / M_{\mathrm{pl}}^{4 / 3} \ll 1$. Thus, expanding in $\delta$, the leading order result is obtained by setting
$N_{X}^{\prime} \approx 0$, which is the equilibrium condition before the recoupling due to pocket contraction.

Given the above assumptions, before the annihilation process recouples, we have the quark number conservation $N_{1}=N_{q}^{\text {initial }}-2 N_{2}-3 N_{3}$. Applying the equilibrium condition before recoupling and neglecting the escape and annihilation terms for the bound states at that point gives

$$
\begin{aligned}
2\langle\sigma v\rangle_{11 \rightarrow 20}\left(N_{1}^{2}-\tilde{f}_{1} N_{2} V\right) & =\langle\sigma v\rangle_{21 \rightarrow 30}\left(N_{2} N_{1}-\tilde{f}_{2} N_{3} V\right), \\
\langle\sigma v\rangle_{21 \rightarrow 30}\left(N_{1} N_{2}-\tilde{f}_{2} N_{3} V\right) & =\langle\sigma v\rangle_{3(-1) \rightarrow 20}\left(N_{3} N_{1}-\tilde{f}_{3} N_{2} V\right),
\end{aligned}
$$

where

$$
\begin{aligned}
& \tilde{f}_{1}=\frac{\left(n_{1}^{e q}\right)^{2}}{n_{2}^{e q}} \propto \exp \left(-\Delta E_{1} / T_{c}\right), \\
& \tilde{f}_{2}=\frac{n_{2}^{e q} n_{1}^{e q}}{n_{3}^{e q}} \propto \exp \left(-\Delta E_{2} / T_{c}\right), \\
& \tilde{f}_{3}=\frac{n_{3}^{e q} n_{1}^{e q}}{n_{2}^{e q}} \propto \exp \left(-\Delta E_{3} / T_{c}\right),
\end{aligned}
$$

and $\Delta E_{i}$ denote the heat released during the above processes. The solution to the above algebraic set of equations provides the abundances of quarks and bound 
states in the contracting pocket before recoupling as a function of the pocket radius $R$. The total abundance of the produced color-singlet baryons is given by the total baryon abundance $N_{3}$ evaluated at the recoupling radius $R_{\text {rec }}$. Notice that $e^{-\Delta E_{1,2} / T_{c}} \sim e^{-\alpha^{2} m_{q} / T_{c}} \gg e^{-\Delta E_{3} / T_{c}} \sim e^{-m_{q} / T_{c}}$, where $\alpha$ is evaluated at the bound state's Bohr radius. Thus, we identify a strong hierarchy $\tilde{f}_{1}, \tilde{f}_{2} \gg \tilde{f}_{3}$.

As a result, a simple analytic expression for the baryon fraction that survives the phase transition relative to the initial quark abundance $N_{q}^{\text {initial }}$ can be found. In the limit of inefficient bound state breaking reactions $\tilde{f}_{1,2} V \ll 1$, it is

$$
\frac{N_{3}}{N_{q}^{\text {initial }}}=\frac{2\langle\sigma v\rangle_{21 \rightarrow 30}\langle\sigma v\rangle_{11 \rightarrow 20}}{\langle\sigma v\rangle_{3(-1) \rightarrow 20}\langle\sigma v\rangle_{21 \rightarrow 30}+4\langle\sigma v\rangle_{3(-1) \rightarrow 20}\langle\sigma v\rangle_{11 \rightarrow 20}} .
$$

Thus, assuming all the cross sections are of the same order of magnitude, we see that the baryon survival factor is of order one, if deeply bound states dominate the system. This is the case if the scale hierarchy $m_{q} \gg \Lambda$ is taken to be extremely large.

In the regime of efficient bound state breaking $\tilde{f}_{1,2} V \gg 1$, we find stronger DM abundance suppression. To simplify things even further, we assume that the terms with $\tilde{f}$ dominate and that $\tilde{f}_{1} \sim \tilde{f}_{2} .{ }^{8}$ With these assumptions, we find that at the recoupling radius, we have

$$
\frac{N_{3}}{N_{q}^{\text {initial }}}=\frac{4 \pi v_{w}^{3}}{3 \tilde{f}_{1}^{2} N_{q}^{\text {initial }}\langle\sigma v\rangle_{1(-1) \rightarrow 00}^{3}} .
$$

Now if we assume in Eq. (3.12) the integral is dominated by the contribution around the recoupling point where the bound state total numbers peak, we find

$$
\mathcal{S}_{\text {symm }} \approx 9 \frac{v_{q}}{v_{w}} \frac{4 \pi v_{w}^{3}}{3 \tilde{f}_{1}^{2} N_{q}^{\text {initial }}\langle\sigma v\rangle_{1(-1) \rightarrow 00}^{3}} .
$$

We can better understand from this equation the effects that various parameters have on the survival factor. Increasing the quark velocity $v_{q}$ enhances their escape rate [see Eq. (3.4)], thus increasing $\mathcal{S}_{\text {symm. }}$. We also see that by increasing $\langle\sigma v\rangle_{1(-1) \rightarrow 00}$, the survival factor decreases, which was expected since, by increasing this cross section, quarks become more likely to annihilate against each other than bind together. For shallower bound states, the binding processes are less favored; thus, we expect that the survival factor should decrease. This is exactly what Eq. (3.20) suggests: For shallower bound states, the Boltzmann

\footnotetext{
${ }^{8}$ Both $\tilde{f}_{1}$ and $\tilde{f}_{2}$ depend on the ratio $m_{q} / \Lambda$. For $m_{q} / \Lambda \lesssim 1000$, they are within an order of magnitude of each other, justifying our assumption. Neglecting this difference allows us to find a simple analytic formula that sheds light on the effect of various quantities on the survival factor.
}

suppression in $\tilde{f}_{1}$ becomes less severe and $\tilde{f}_{1}$ increases; thus, $\mathcal{S}_{\text {symm }}$ decreases.

The initial density of quarks in a pocket is determined via a preconfinement, perturbative freeze-out calculation. Yet, $N_{q}^{\text {initial }}$ in Eq. (3.20) depends on the initial pocket radius, too. Thus, through $N_{q}^{\text {initial }}$ we find that $\mathcal{S}_{\text {symm }} \sim R_{i}^{-3}$.

Finally, by decreasing $v_{w}$, according to Eq. (3.15), the recoupling radius increases, which gives less time for the baryon abundance in the pocket to build up before the interactions become efficient again; see Fig. 6. A larger recoupling radius means a smaller peak value for the $N_{3}$ abundance, like the one seen at $R \Lambda \sim 10^{5}$ in Fig. 6, which in turn decreases the survival factor $\mathcal{S}_{\text {symm }}$. This behavior is exactly what we see in Eq. (3.20).

\section{Effect of quark pressure and summary of assumptions}

The $v_{w}$ scaling of Eq. (3.20) helps us better understand how our determination of $\mathcal{S}$ would change had we included the effect of quark pressure on $v_{w}$. This equation suggests that by using Eq. (2.9) for $v_{w}$ and ignoring the fact that quark pressure can oppose pocket contraction, we are actually calculating an upper bound on the survival factor since we are certainly overestimating $v_{w}$. Also, this $v_{w}$ scaling, combined with the proximity of $\mathcal{S}_{\text {symm }}$ to the asymmetry bound $\eta_{\text {rms }}$ across our parameter space when we use Eq. (2.9), motivates us to believe that when the quark pressure is properly taken into account, we should expect that the asymmetry bound $\mathcal{S}=\eta_{\text {rms }}$ is saturated for every point in the parameter space that we study (see Appendix A 4 for more empirical evidence of this claim). In the next section, we use these two limits to bracket the parameter space of the model that reproduces the observed DM relic abundance. We refer to these two limiting scenarios as the zero quark pressure and the asymmetry scenarios.

Before solving the Boltzmann equations, it is useful to review all the parameters affecting our calculation of $\mathcal{S}$ and the final DM abundance. The UV model has a very limited set of parameters: the confinement scale $\Lambda$ and the quark mass $m_{q}$. These parameters feed into the calculation of a few secondary quantities that directly affect the calculation of $\mathcal{S}$ and are listed in Table II. A precise calculation of these secondary quantities requires various nonperturbative studies. These quantities can be divided into two broad categories: macroscopic and microscopic.

The macroscopic quantities are those concerning the dynamics of the bubbles and pockets, i.e., their initial radius $R_{i}$ and their wall velocity $v_{w}$. While our expressions for these quantities in Eqs. (2.8) and (2.9) were based on a simplified simulation of the phase transition (see Appendix A), there is extensive literature concerned with the detailed calculation of these quantities. Unfortunately, this literature has not yet settled on a single, definitive calculation of these quantities, which is why we content ourselves with simple order-of-magnitude estimates. (See, 
for example, Refs. [63,67-69] and references within for various calculations of the wall velocity.)

The microscopic quantities include various cross sections and binding energies. They also determine the dimensionless interquark spacing $\xi$, which directly affects our final results as well. We use the results from [61] for the cross sections and the binding energies. We summarize the relevant quantities in Appendixes B and C.

It is also worth reiterating a few important assumptions that significantly streamlined our analysis. Recall that in Sec. II B, we argued that the wall velocity is controlled by the amount of supercooling and quark pressure during the phase transition. Following that assumption, we found that the typical velocity of quarks in Eq. (2.11) is much faster than the wall velocity even when the quark pressure effect is neglected in Eq. (2.9). Therefore, any density gradient within a pocket caused by the compression of the walls can be quickly smoothed out by the thermal motion of the quarks. As a result, we assume that the particles within the pockets are homogeneously distributed, which simplifies our analysis significantly.

We also neglect the abundance of the bound states before the phase transition. Furthermore, as suggested in Fig. 1, we assume the quarks are initially well separated inside the pockets and that they promptly rebound off the wall surface. As we will argue later, all of our assumptions determine the parts of the parameter space where our analysis is valid.

In principle, the baryons formed could further interact to form dark nuclei at the end of the pocket contraction phase. The formation of such multibaryon states in dark sectors has been discussed in Ref. [70]. To estimate the effect in our scenario, we can rescale the predicted interaction rate by the compression factor inside the pockets, which

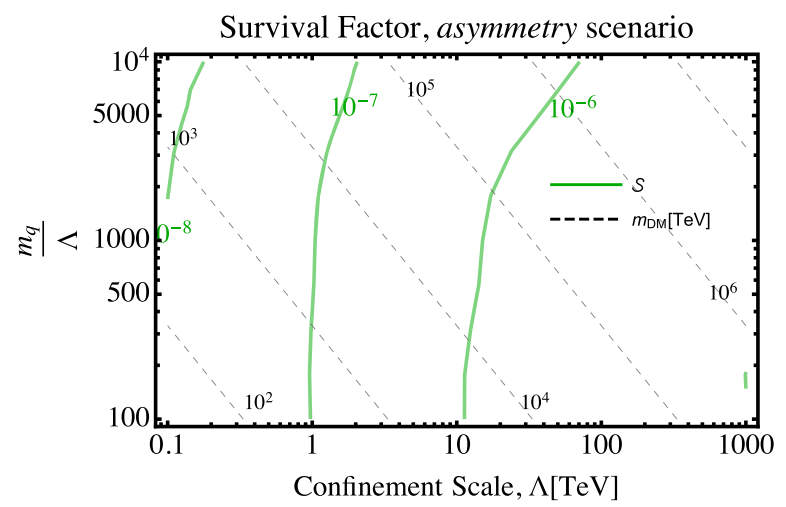

quadratically enhances the formation rate. The contraction factor is defined as $c_{f}=\left(R_{1} / R_{\text {rec }}\right)^{3}$, where $R_{\text {rec }}$ is the radius where the interactions recouple. An estimate of this value based on our analytic solution gives $c_{f} \approx 10^{4}\left(m_{q} / \Lambda\right)^{3 / 2}$. In addition, the number density of

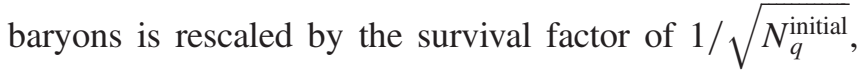
which counteracts the compression effect. Evaluating this in our scenarios, where the constituent quark mass is large, and thus the number density at freeze-out is significantly reduced, leads to the conclusion that, in the parameter space region we consider, the dibaryon fraction is at most of the order of $0.5 \%$. Formation of larger multibaryon clusters is also severely suppressed due to this dibaryon production bottleneck.

\section{E. Results and discussion}

We now turn to the central results of this paper. We scan over a range of $\Lambda$ and $m_{q} / \Lambda$ values, solving the Boltzmann equations at each point to calculate the survival factor $\mathcal{S}$. As mentioned above, we use Eq. (2.9) for the wall velocity when solving the equations and finding the viable part of the model's parameter space that produces the correct present-day abundance of DM. We argued that this zero quark pressure scenario and the asymmetry scenario, in which we assume $\mathcal{S}=\eta_{\mathrm{rms}}$, are the two limiting cases that bracket the uncertainties in our DM relic abundance calculation. We find that these two scenarios only give rise to an $\mathcal{O}(1)$ difference in the DM mass range that can explain the observed relic abundance.

In Fig. 7, we show contours of a constant survival factor for both of these scenarios and for different values of $\Lambda$ and $m_{q} / \Lambda$. The asymmetry scenario plot shows the smallest

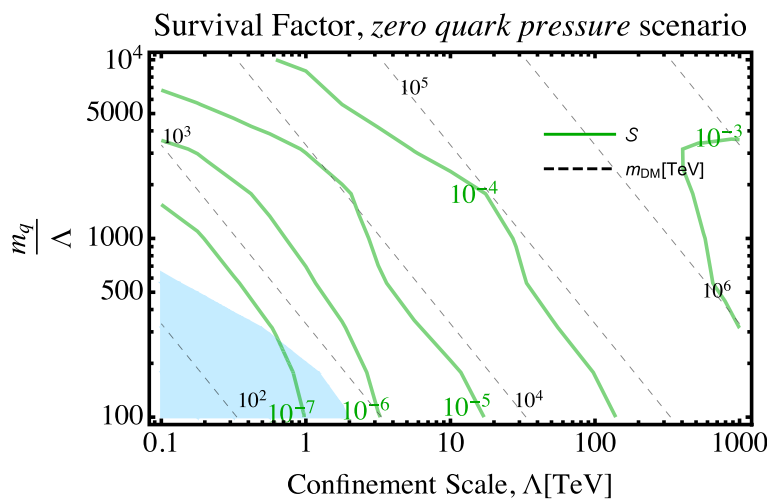

FIG. 7. Contours of constant survival factor $\mathcal{S}$ (green contours) in the two limiting scenarios that we consider: (i) assuming the asymmetry bound on $\mathcal{S}$ is saturated (on the left) or (ii) neglecting the quark pressure effect on the pocket wall velocity (on the right). The contours of constant DM mass in TeV are shown as well (black dashed). To obtain the right plot, we solve the full set of Boltzmann equations in Eq. (3.10) using the values for the initial pocket radius $R_{i}$ and the pocket wall velocity $v_{w}$ based on our simulation results discussed in Sec. II and Appendix A. In the light blue region on the right plot, we find that the suppression is so severe that only the accidental asymmetric abundance of quarks in each pocket survives after the phase transition. For the left plot, we simply assume $\mathcal{S}=\eta_{\text {rms }}$ for every point in the parameter space. We observe orders-of-magnitude suppression in the DM abundance due to the second stage of annihilation during the phase transition in either scenario. Note that the small difference in the $10^{-7}$ contours in the overlap region is a plotting artifact. 
survival factor possible while the zero quark pressure scenario gives an upper bound on the survival factor for every point in the parameter space; see the discussion in Sec. III C. In the asymmetry scenario, the only sources of uncertainty are those affecting the preconfinement calculation and the initial pocket size, while in the zero quark pressure scenario, the uncertainty in determining the wall velocity $v_{w}$ should also be included.

The available parameter space in the asymmetry limit scenario is shown in Fig. 8. Equation (2.7) shows that as $\Lambda$ increases, $R_{1}$, and thus the number of trapped quarks inside the pocket, decreases. Thus, as expected from Eq. (2.16), we find that the larger the initial radius, the smaller the survival factor.

We should keep in mind that many simplifying approximations were made about the dynamics of the phase transition in Appendix A in order to obtain Eq. (2.8) for the bubble radius. This, inevitably, introduces some uncertainty in our calculation. To characterize this uncertainty, in Fig. 8 we introduce a fudge factor for the bubble radius denoted by $f_{R}$, to be multiplied against the values from Eq. (2.8). The observed relic abundance line moves within the light purple band as we vary $f_{R}$ between 0.1 and 10 . Any point

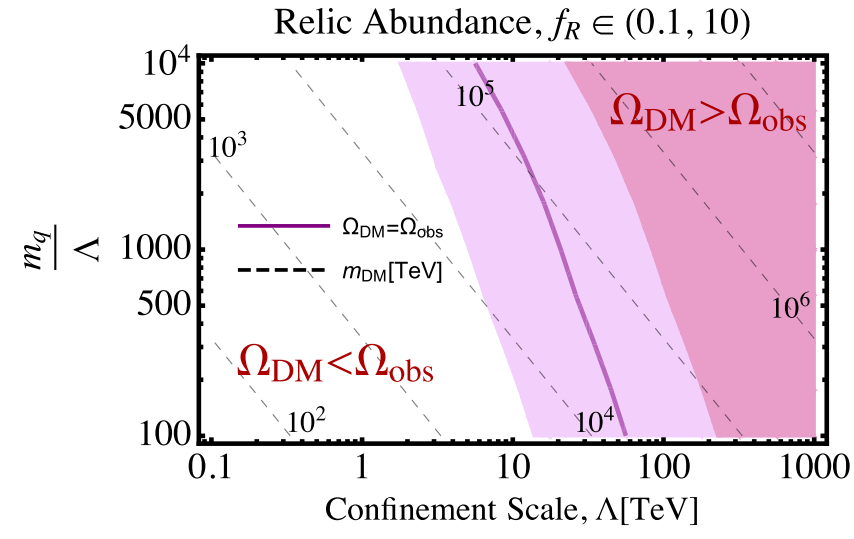

FIG. 8. Produced abundance of dark baryons, the DM candidate, in the asymmetry abundance scenario. The black dashed lines are contours of constant DM mass in $\mathrm{TeV}$. The relic abundance line (with the initial radius fixed to its central value, i.e., $f_{R}=1$ ) is plotted (purple line) along with its uncertainty (light purple shades) corresponding to an order-of-magnitude variation of the initial radius. The shaded red region is excluded, as it produces too much DM, while the unshaded region produces too little DM. The baryons can therefore constitute a subcomponent of the DM within the unshaded regions of parameter space. The survival factor is determined by the accidental asymmetry of the pocket, which is independent of the wall velocity as long as the asymmetry bound is saturated. Thus, the major source of uncertainty in the location of the relic abundance line is the initial pocket radius. We also find that the uncertainty from microscopic quantities is subdominant to those of the initial pocket radius. This figure clearly shows that the baryon masses accounting for the observed DM abundance can be much heavier than the unitarity bound [53]. above and to the right of the relic abundance line, including the entire red region, is ruled out.

Since the asymmetry limit scenario is the lowest attainable $\mathcal{S}$ in our setup, the relic abundance line in this scenario is an upper bound on the possible masses in our model.

In the other limit, the zero quark pressure scenario provides us with a lower bound on the range of DM masses in this setup that can explain the observed DM abundance. In Fig. 9, we show the available parameter space in this scenario. The calculation can now be affected by a change in both the initial pocket radius $R_{1}$ and its wall velocity $v_{w}$. To characterize this uncertainty, in Fig. 9 we introduce a fudge factor for both the bubble radius and the wall velocity, denoted by $f_{R}$ and $f_{v}$, respectively. As expected, for any fixed $\Lambda$ the observed DM relic abundance in this scenario is obtained by smaller DM masses than that of the asymmetry scenario in Fig. 8.
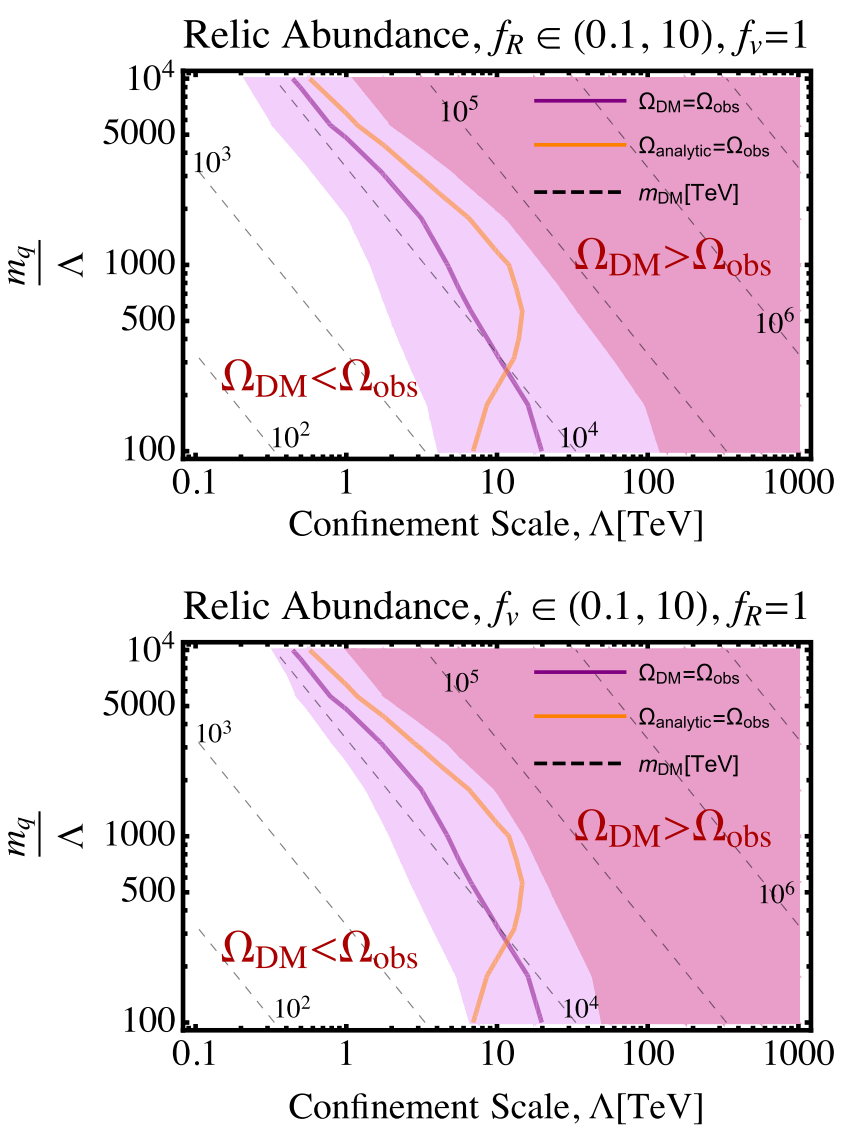

FIG. 9. Similar to Fig. 8 but now with zero quark pressure. We vary the pocket initial radius (top) or the wall velocity (bottom) within 1 order of magnitude of the central values in Eqs. (2.8) and (2.9) to characterize the uncertainty in the final relic abundance calculation stemming from these quantities. The relic abundance line using the analytic approximation of Eq. (3.20) for the survival factor is denoted by the orange curve, too. Even with the quark pressure neglected, we still find a substantial suppression in the DM abundance during the phase transition. We also find that the baryon masses accounting for the observed DM abundance can be much heavier than the unitarity bound [53]. 
In this figure, we also show the relic abundance line when we use the analytic approximation of Eq. (3.20) to calculate the survival factor. We find reasonable agreement between our analytic approximation (orange curve) and the full numerical result (purple curve).

In both of these limiting scenarios studied in Figs. 8 and 9, we find a similar range of DM masses and $\Lambda$ that can account for the present-day DM abundance. We expect that these two scenarios bracket the true location of the relic abundance line when the effect of the quark pressure on the pocket wall velocity is appropriately included. The figures indicate that, depending on the macroscopic parameters, the region of parameter space that produces the observed DM abundance predicts $m_{\mathrm{DM}} \sim \mathcal{O}(1)-\mathcal{O}(100) \mathrm{PeV}$, well above the thermal relic unitarity bound of $m_{\mathrm{DM}} \lesssim 300 \mathrm{TeV}$ [53]. Even with various sources of uncertainty, our results predict a confinement scale roughly in the $\mathcal{O}(1)-$ $\mathcal{O}(100) \mathrm{TeV}$ range, in contrast to [25], which predicts a much wider range of confinement scales in such models. The parameter space above this range is ruled out, while the remaining parameter space is allowed, producing a subcomponent of DM.

The values of the cross sections and the binding energies entering the Boltzmann equations can be found in Appendixes B and C, respectively. We find that the uncertainty in our results due to these microscopic quantities is subdominant to the uncertainty from the macroscopic bubble dynamics parameters discussed above. For further details about these parameters and the uncertainties in determining them, see the aforementioned Appendixes and the references therein.

Determining the exact position of the relic abundance line requires more precise calculations of both macroscopic and microscopic quantities. Nonetheless, such calculations will not change our qualitative conclusion: that the phase transition gives rise to a new stage of annihilation that reduces the relic abundance by orders of magnitude and shifts the DM mass to well above the unitarity bound.

We can also understand the expected results for the parts of parameter space not plotted. For larger $\Lambda$ s than were plotted, Figs. 8 and 9 suggest that this model always overproduces $\mathrm{DM}$ and is ruled out. For smaller $\Lambda \mathrm{s}$ than were plotted, our assumption that the preconfinement abundances of bound states are negligible breaks down. Since so many baryons are produced before the start of the phase transition, the survival factor becomes comparable to 1 . For low enough $\Lambda$, we should use the combinatoric calculation of the relic abundance described in Ref. [25]. An additional aspect is that, in this region of parameter space, the glueball lifetime can be substantial, which leads to entropy injection and makes the abundance calculation more model dependent, as discussed explicitly for several models in Ref. [25]. Further investigation of this region is left for future works.

As we go to larger values of $m_{q} / \Lambda$, our assumption that $v_{q} \gg v_{w}$ breaks down. In this case, local inhomogeneities appear in the distribution of particles in the pockets, and the entire homogeneous system of equations in Eq. (3.10) must be modified. Furthermore, we find that, for higher $m_{q} / \Lambda$ than is shown in Figs. 8 and 9, the quark separations during the contraction epoch can become as low as $\sim 1 / \Lambda$ (due to the small cross sections allowing for a greater degree of compression). In this case, the picture of well-separated quarks that rebound off the stiff bubble wall (before they run into other colored particles) must be modified. Nonperturbative effects become more relevant in this case. It is also possible that at such high densities, quarks bind into more stable and massive dark nuclear states such as nuggets; see [71] for a study of dark quark nuggets in the light dark quark limit.

Furthermore, a recent study has investigated the creation of multibaryon bound states in a very similar framework [72]. It was found that at $m_{q} / \Lambda>10^{8}$ the fermi pressure of the quark constituents can lead to the formation of compact objects. However, the framework of the mentioned investigation significantly differs from the setup used in our work. In particular, the DM number density is fixed by a preexisting asymmetry, and the temperature of the decoupled dark sector is chosen as an initial condition.

Finally, as we go to lower values of $m_{q} / \Lambda$, eventually the first-order phase transition turns into a second-order one and then a cross-over; see e.g., [41]. In this regime there will be no bubble walls to compress quarks into a second stage of annihilation. Even for lower values of $m_{q} / \Lambda$ for which there still exists a first-order phase transition, we run the risk of breaking our assumption that the string breaking rate is negligible, so quarks and diquarks can escape from the pocket before significant annihilation takes place.

All in all, outside of the window shown in Figs. 8 and 9, either the parameter space is already ruled out, or at least one of the simplifying assumptions we made fails and our analysis becomes unreliable.

\section{F. Extensions of our analysis}

So far we have focused on a confining $S U(3)$ gauge group with a single generation of heavy fermions in the fundamental representation. Nonetheless, it is conceptually straightforward to repeat our analysis for slightly different setups. In this section we comment on the differences that we expect would arise had we varied the number of colors, $N_{c}$, or the quark representation.

Had we chosen a gauge group with a larger number of colors, $S U\left(N_{c} \geq 4\right)$, we expect that we would have found a smaller $\mathcal{S}_{\text {symm }}$ since the stable DM candidate in such a theory (the analogue of the baryon) requires more constituent quarks to bind together in more steps. (Notice that in Fig. 6 as the quark number of a state increases, its abundance decreases within a pocket.) However, if even with $N_{c}=3$ we find $\mathcal{S}_{\text {symm }} \leq \eta_{\text {rms }}$, we expect to saturate the asymmetry bound for larger gauge groups as well. The additional $\mathcal{S}_{\text {symm }}$ suppression would not change the final survival factor $\mathcal{S}$. 
The quark representation under the dark gauge group has a slightly more complicated effect on our results. For any quark representation, one must first identify the list of all possible bound states and then write down the Boltzmann equations with all possible interactions. As explained in Sec. III C, the binding energies of these bound states can also have a significant effect on the solutions of the Boltzmann equations. As an example, consider the case in which quarks are in the adjoint representation of the group. These quarks can bind with gluons to form colorneutral gluequarks [27]. Since the gluons can be found abundantly, we expect that quarks can easily pass through pocket walls by binding with a nearby gluon. Thus, pocket walls will not compress the quarks, and there will be no second stage of annihilation due to the phase transition.

Besides changing the model under consideration, our work would also benefit from improving our orderof-magnitude estimates and simplifying assumptions. Dedicated numerical simulations that more carefully model the bubble dynamics and nonperturbative physics could reduce the uncertainties in both macroscopic and microscopic quantities listed in Table II, narrowing down the uncertainty on the relic abundance line in Figs. 8 and 9.

\section{POTENTIAL EXPERIMENTAL SIGNALS}

So far, our study has only relied on fairly general properties of a dark sector. We assumed that the dark sector under study is a confining $S U(3)$ gauge theory with a single generation of heavy fermions; we also assumed a portal exists between the sectors that keeps them in kinetic equilibrium and allows the glueballs and mesons to decay to the SM. All the conclusions drawn in the previous sections were independent of further details of the portal and the origin of the heavy dark quark mass.

A detailed study of all the phenomenological signals of such a sector has to be carried out in a model-dependent way with a specified portal. As a result, here we merely list the signals and constraints that should be expected from this broad class of models.

(i) The main feature of our setup is a first-order phase transition in the early universe. Such a phase transition can also give rise to a stochastic gravitational wave (GW) background that can be detected in a host of different future experiments; e.g., see [73] for a recent study of the GW signals of confining dark sectors. The characteristics of the resulting GW, such as the frequency and the strength, depend on a handful of thermodynamical parameters; see [74] for a brief review. This GW signal is independent of the portal to the SM. A naive estimate ${ }^{9}$ shows that different parts

\footnotetext{
${ }^{9} \mathrm{We}$ use the formulas in [75] to estimate the GW signal produced during the phase transition. We use the interface introduced in [56] to compare the result to the reach of various experiments.
}

of our parameter space could potentially be probed in future experiments like DECIGO [76,77] and BBO [78]. Early universe phase transitions can also give rise to anisotropies in the GW spectrum, which can potentially be detected at future facilities (see e.g., [79]). Given the extremely high mass range of the DM candidates in our model, the GW signals could have the highest discovery potential in such sectors. We leave the further study of GW signals in this class of models for future work.

(ii) The glueballs and the mesons are unstable due to the portal to the SM. Stringent bounds from BBN require that these relics have a short lifetime. See for instance [80-82] for recent studies. As a rule of thumb, one can avoid various constraints by assuming all these bound states decay before the BBN; i.e., their lifetime is $\tau \leq 1 \mathrm{~s}$. This bound on the lifetime introduces a lower bound on the strength of the portal. This lower bound can vary substantially depending on the details of the portal. Our requirement that both sectors are in kinetic equilibrium also imposes a lower bound, though we expect the BBN bound to be more stringent.

(iii) The portal to the SM introduces possible direct and indirect detection signals. However, the DM number density in the Universe and in our Galaxy is very suppressed due to this model's heavy DM mass. A naive estimation suggests that our model's indirect signal from DM annihilation within the Milky Way is severely suppressed and undetectable. The direct detection signal, however, depends on the details of the portal and should be studied model dependently. We note that in this heavy mass range, even very large DM-SM elastic cross sections are allowed, but within the reach of upcoming and ongoing experiments [83].

(iv) A separate indirect detection signal comes from the observation that our composite DM model admits excited states. Deexcitations from these excited states might lead to radiation that could be detected. Excitations could be produced in the early universe or via interactions with matter today.

(v) Yet another indirect signal could come from the capture of DM in celestial bodies; see for example Refs. $[84,85] .{ }^{10}$ As DM accumulates at the bottom of these potential wells, it can begin to annihilate at a significant rate, possibly affecting the evolution of these celestial bodies in an observable way or enhancing a potential annihilation signal $[88,89]$.

(vi) For the $\mathcal{O}(\mathrm{PeV})$ and above $\mathrm{DM}$ masses predicted in our model, direct production of DM at collider facilities is not possible in the foreseeable future.

\footnotetext{
${ }^{10}$ See also $[86,87]$ for studies of lighter DM capture in gravitational basins or exoplanets, respectively.
} 
Yet, if the portal is substantially lighter, it can be directly observed at collider experiments. While the dark quarks are too heavy to produce at collider facilities, the glueballs of the new dark sector, whose mass is $\mathcal{O}(10 \Lambda)$, could potentially be produced at future colliders.

(vii) Various studies suggest an upper bound on the DM self-scattering [90-96]. As a rough estimate,

$$
\sigma_{\mathrm{SI}} / m_{\mathrm{DM}} \lesssim 1 \mathrm{~cm}^{2} \mathrm{~g}^{-1} \sim(60 \mathrm{MeV})^{-3} .
$$

It is straightforward to check that for the high confinement scales we are studying, this upper bound is easily satisfied.

(viii) One can also search for signals coming from the inhomogeneities in the DM density that were produced during the phase transition when DM was compressed by contracting pockets, but this seems unlikely. By performing a Jeans stability analysis, we find that the internal baryon pressure easily overcomes the self-gravity of these overdensities. Therefore, pockets do not seed self-gravitating DM clumps. One might also look for modifications to the matter power spectrum due to these overdensities, but initial estimates indicate that the matter power spectrum would only be modified at unobservably small mass scales if at all. Specifically, the total DM mass within a horizon radius soon after the phase-transition epoch (after which the comoving abundance is fixed) can be estimated as the DM density multiplied by $\mathrm{H}^{-3}$, with a DM density crudely approximated (ignoring changes in the number of relativistic degrees of freedom over time) as $\sim\left(\Lambda / T_{\mathrm{CMB}, 0}\right)^{3} \times$ the present cosmological density of DM, where $T_{\mathrm{CMB}, 0} \sim 2 \times 10^{-4} \mathrm{eV}$ is the presentday temperature of the radiation bath. This gives an enclosed mass:

$$
\begin{aligned}
M_{\mathrm{enc}} & \sim\left(\Lambda^{2} / M_{\mathrm{pl}}\right)^{-3}\left(\Lambda / T_{\mathrm{CMB}, 0}\right)^{3} \times 10^{-6} \mathrm{GeV} / \mathrm{cm}^{3} \\
& \sim\left(\frac{1 \mathrm{TeV}}{\Lambda}\right)^{3} \times 10 \mathrm{~kg} .
\end{aligned}
$$

Thus, for phase transitions at the $\mathrm{TeV}$ scale and above, we would expect phase-transition-induced inhomogeneities to affect DM clumps at the $\mathrm{kg}$ scale and below. Even if these clumps survived, this mass scale is vastly lower than what can be probed by any possible observational constraints on the matter power spectrum, which are currently exploring halo masses of order $10^{7-8} M_{\odot}$ (e.g., [97-99]).

Because of its low number density, the dark matter in our setup can have significant interactions with the SM particles and still have escaped detection so far. Creative new search strategies will be needed to explore this possibility. Novel ideas for direct detection of such a scenario have been put forward in Ref. [83], and interesting signals in heavy isotope searches [100] could arise if our dark baryons can bind to SM atoms and nuclei.

In addition to the above signals, which should exist for any specific realization of the DM-SM portal, there may exist additional portal-dependent signatures. We also find, using the results of Ref. [25], that depending on the type of portal to the SM, the glueballs' lifetime could be larger than the Hubble time at $T=\Lambda$. In such a scenario, the delayed decay of the glueballs can further dilute the DM abundance $[27,30]$ in the parameter space that we have studied, thus pushing the relic abundance line in Figs. 8 and 9 to even higher DM masses. A proper study of this effect, as well as other signals from any specific portal, is left for future works.

\section{CONCLUSION}

In this work we studied the consequences of a first-order phase transition in a confining dark sector with a single heavy quark in the fundamental representation. We assumed a portal exists between our sector and the dark sector that keeps the two sectors at kinetic equilibrium at the time of the phase transition and respects dark baryon number conservation. The arguments we presented do not depend on further details of the portal.

We argued that the bubbles of the confined phase, after nucleation, expand very slowly. Soon after the bubbles come in contact and coalesce, pockets of the deconfined phase form and are submerged in a sea of the confined phase. The quarks are trapped inside these isolated and ever-contracting deconfined phase pockets. There is always an accidental asymmetry in the net dark baryon number in a given pocket, due to local stochastic fluctuations in the number of quarks and antiquarks at the onset of pocket formation. As the pockets contract, the enclosed quarks compress until formerly frozen-out interactions recouple, giving rise to a second stage of annihilation.

We wrote down the complete set of Boltzmann equations with all $2 \rightarrow 2$ interactions between lowest-lying bound states of the heavy quark. By solving these equations, we were able to calculate the fraction of dark quarks that survive the second annihilation event. These surviving quarks bind into stable, color-singlet states that comprise the DM abundance we see today. We find that these Boltzmann equations predict a dramatic suppression in the DM relic abundance. This suppression is sensitive to the initial size of the pocket, the density of the quarks trapped within, and the pocket wall velocity. While there is a large uncertainty in determining these parameters, we showed that for virtually any reasonable values of these parameters, there is a significant suppression in the DM relic abundance.

We find the effect of quark pressure on the pocket wall velocity difficult to model. However, we do know that this 
effect will further slow down the pocket wall, which we implies a smaller survival factor. We calculated the relic abundance of DM in this setup in two extreme scenarios: (i) in the zero quark pressure scenario and (ii) when we assume the quark pressure is so severe that the asymmetry bound on the survival factor is saturated. These two limiting scenarios bracket the range over which the relic abundance line can move when the quark pressure effects are properly taken into account. We found that for a fixed dark confinement scale $\Lambda$, the DM mass in this setup only changes by $\mathcal{O}(1)$ factors between these two scenarios.

After identifying the parts of the $m_{q}-\Lambda$ parameter space that predict the observed present-day DM abundance, we found that this large suppression opens up parts of the parameter space that were previously thought to be ruled out. In particular, we found a DM mass scale well above the often-quoted unitarity bound. Our calculation also suggests an upper bound on the dark confinement scale $\Lambda \sim \mathcal{O}(1)-\mathcal{O}(100) \mathrm{TeV}$. For any $\Lambda$ above this bound, DM is overproduced, despite the dramatic suppression of its abundance during the phase transition. Depending on the value of $\Lambda$, the dark baryon mass that can explain the observed DM abundance varies roughly between $10^{3}$ and $10^{5} \mathrm{TeV}$.

There are many possible signals that our setup can give rise to. With the exception of gravitational waves, all the other potentially detectable signals depend on the specific form of this model's portal to the SM. It will be interesting to investigate the signatures of specific portals and their constraints, which we leave to future work.

There are numerous ways in which our analysis can be improved. To decrease the uncertainties in our results, it will be important to perform more detailed numerical simulations of the macroscopic bubble dynamics during the phase transition and the microscopic strong dynamics that determine the particle interactions. The most important quantities to be calculated would be the initial pocket size and its subsequent contraction rate, and the cross sections and binding energies included in our Boltzmann equations. Additionally, it will also be interesting to study the relic abundance calculation for other gauge groups and different quark representations. Furthermore, for any specific portal, we should study the potential DM dilution due to a delayed glueball decay after the phase transition.

Confining sectors are natural dark sector candidates. In this paper we focused on such sectors with only a single species of heavy dark quark. We pointed out the dramatic effect that this model's first-order phase transition has on the relic DM abundance of such a sector. The dynamics lead us to a sharp prediction about the natural mass scale of such DM candidates, $10^{3-5} \mathrm{TeV}$. It is of paramount importance to study variations of these minimal dark sectors in greater detail and their potential signatures in various upcoming experiments.

\section{ACKNOWLEDGMENTS}

We thank Andrei Alexandru, Tom Cohen, Daniel Hackett, Julian Muñoz, Jessie Shelton, and Xiaojun Yao for useful discussions. We especially thank Benjamin Svetitsky for his collaboration in early stages of this work. We also thank Yonit Hochberg, Graham Kribs, Rebecca Leane, Michele della Morte, Michele Redi, and Kai Schmidt-Hoberg for constructive comments on the draft. In particular, we thank Filippo Sala for raising the question of quark pressure effects. The work of P. A., G. W. R., and T.R.S. was supported by the U.S. Department of Energy, Office of Science, Office of High Energy Physics, under Contract No. DE-SC0012567. The work of P. A. was also supported by the MIT Department of Physics. G. W. R. was also supported by a National Science Foundation Graduate Research Fellowship and the U.S. Department of Energy, Office of Science, Office of Nuclear Physics under Contract No. DE-SC0011090. The work of E. $\mathrm{K}$. was supported by the Israel Science Foundation (Grant No. 1111/17), by the Binational Science Foundation (Grant No. 2016153), and by the I-CORE Program of the Planning Budgeting Committee (Grant No. 1937/12). The work of E. D. K. was supported by the Zuckerman Science, Technology, Engineering, and Mathematics Leadership Program and by ISF and I-CORE grants of E. K. J. S. was largely supported by a Feodor Lynen Fellowship from the Alexander von Humboldt Foundation.

\section{APPENDIX A: THERMODYNAMICS OF A FIRST-ORDER PHASE TRANSITION}

In this Appendix, we collect results that are helpful for understanding the dynamics of a first-order phase transition. We also describe a numerical simulation of the confining phase transition from the main text, which we perform to fix the initial pocket radius and its contraction rate. The code we used to perform this simulation can be found in Ref. [112].

\section{Standard thermodynamics}

The defining characteristic of a first-order phase transition is that a first derivative of the free energy, or the free energy density $f$, is discontinuous at a critical point. In our case, the entropy density, $s=-\frac{\partial f}{\partial T}$, is discontinuous, corresponding to a nonzero latent heat release due to phase conversion. In contrast, $f$ itself is continuous, meaning that the free energy of the two phases is the same at the critical point, $f_{\text {deconf }}=f_{\text {conf }}$.

To better understand the latent heat, first notice that in the absence of chemical potentials, the critical point is specified by a single parameter, the critical temperature $T_{c}$, and the free energy density is minus the pressure, $f=-p$. Since the free energy of both phases is the same at the critical point, their pressure is the same. Using the Euler relation, $\rho=T s-p$, where $\rho$ is the energy density, one can take the difference in energy densities of the two phases at the critical temperature to find 


$$
\Delta \rho=T_{c} \Delta s \equiv l
$$

which defines the latent heat density. Whereas the free energy density is continuous, the energy density is not. Another important quantity that characterizes first-order phase transitions is the surface tension $\sigma$. The surface tension is the energy cost per unit area of an interface separating the two phases.

The latent heat density and surface tension are calculable via lattice simulations. We assume that at temperatures below the quark mass, the thermodynamics of the system are insensitive to the heavy quark field, and we need only consider lattice simulations of pure $S U(3)$ Yang-Mills theory. In particular, the authors of [43] calculated a latent heat density and surface tension in the infinite volume limit (see their Tables 7 and 15),

$$
\begin{aligned}
l & =1.413 T_{c}^{4}, \\
\sigma & =0.02 T_{c}^{3} .
\end{aligned}
$$

These quantities have been computed elsewhere [101-104], and we find that the uncertainties on $l$ and $\sigma$ among these different lattice calculations are not large enough to qualitatively change our results.

As the universe expands, our thermal system, still in the deconfined phase, supercools. ${ }^{11}$ Once the system is supercooled, the free energy of the confined phase is lower than the free energy of the deconfined phase, meaning that it is energetically favorable for a phase transition to take place. For any nonzero amount of supercooling, there now exists a critical radius $R_{c}$ at which the energy cost of increasing a spherical bubble's surface area is exactly compensated by the free-energy decrease due to phase conversion,

$$
\begin{aligned}
& \left.\frac{\partial F}{\partial R}\right|_{R_{c}}=\left.\frac{\partial}{\partial R}\left(4 \pi R^{2} \sigma-\frac{4 \pi}{3} R^{3} \Delta f\right)\right|_{R_{c}}=0 \\
& \Rightarrow R_{c}=\frac{2 \sigma}{\Delta f}
\end{aligned}
$$

where $\Delta f$ is the confined phase free energy density minus that of the deconfined phase, so it is positive.

To relate $\Delta f$ to the latent heat density, first recall that at $T_{c}$ the entropy difference is given by $\Delta s=l / T_{c}$. If we then use the thermodynamic relation $\frac{\partial f}{\partial T}=-s$, we find $\left.\frac{\partial \Delta f}{\partial T}\right|_{T_{c}}=-l / T_{c}$. If we assume small supercooling, we can use a Taylor expansion, which at leading order gives

$$
\begin{aligned}
\Delta f & =\frac{\partial \Delta f}{\partial T}\left(T-T_{c}\right) \\
& =l \frac{\left(T_{c}-T\right)}{T_{c}} .
\end{aligned}
$$

\footnotetext{
${ }^{11}$ Much of the following discussion can be found in Refs. [105] (Sec. 162) and [106] (Secs. 99 and 100).
}

We then find

$$
R_{c}=\frac{2 \sigma T_{c}}{l\left(T_{c}-T\right)} .
$$

The total free energy of a bubble at the critical radius is

$$
\begin{aligned}
F_{c} & =4 \pi R_{c}^{2} \sigma-\frac{4 \pi}{3} R_{c}^{3} \Delta f \\
& =\frac{16 \pi}{3} \frac{\sigma^{3}}{\Delta f^{2}} \\
& =\frac{16 \pi}{3}\left(\frac{\sigma}{T_{c}^{3}}\right)^{3}\left(\frac{l}{T_{c}^{4}}\right)^{-2} \frac{T_{c}^{3}}{\left(T_{c}-T\right)^{2}} .
\end{aligned}
$$

Thermal fluctuations will randomly convert regions of varying shapes and sizes from one phase to the other. When the system is supercooled, there is a chance that a converted region will be large enough that it expands rather than contracts. The probability per unit time and volume of converting a region with free energy $F$ is determined primarily by the Boltzmann factor $e^{-F / T}$. Using dimensional analysis we have [107]

$$
\Gamma=A T^{4} e^{-\frac{F}{T_{c}}},
$$

where $A$ is assumed to be some $\mathcal{O}(1)$ number that is roughly constant with respect to temperature and determined by the microscopic theory. Provided $A$ is indeed an $\mathcal{O}(1)$ number, we find that the exponential is by far the more important factor for determining the behavior of the phase transition, so we set $A$ to 1 without qualitatively changing our results. For an alternative, though similar, expression for $\Gamma$, see [106] (Sec. 99).

Let us make the simplifying assumption that all bubbles can be approximated as spherical. Then, bubbles that nucleate with radii below $R_{c}$ are ephemeral, quickly shrinking due to surface tension, while bubbles with radii well above $R_{c}$ are exponentially less likely to nucleate than critical bubbles by Eq. (A7). Then, to a good approximation, we can assume that only bubbles at the critical radius nucleate. Combining Eqs. (A6) and (A7), we find that the nucleation rate of these critical bubbles is

$$
\begin{gathered}
\Gamma=A T_{c}^{4} e^{-\frac{\kappa T_{c}^{2}}{\left(T_{c}-T\right)^{2}},} \\
\kappa=\frac{16 \pi}{3}\left(\frac{\sigma}{T_{c}^{3}}\right)^{3}\left(\frac{l}{T_{c}^{4}}\right)^{-2}, \\
\sim 7 \times 10^{-5},
\end{gathered}
$$

where in the last line we have used the lattice results from Eq. (A2). Because the latent heat density is an order-one number in units of $T_{c}$ while the surface tension is a small number in units of $T_{c}, \kappa$ turns out to be a very small number 
[which Ref. [43] indicates is generically true for $S U(N)$ theories]. As a result, very little supercooling is required before bubble nucleation becomes efficient.

\section{First half of the phase transition: Bubble growth}

After bubble nucleation begins, the phase transition proceeds via the nucleation of new bubbles and the expansion of old bubbles. To keep track of the progress of the phase transition, we define the fraction of the universe that is in the confined phase [107],

$$
x(t)=\int_{t_{c}}^{t} d t^{\prime} \Gamma\left(t^{\prime}\right) \frac{4 \pi}{3} R^{3}\left(t, t^{\prime}\right)\left(1-x\left(t^{\prime}\right)\right),
$$

where $t_{c}$ is the time at which the universe first reaches the critical temperature and $R\left(t, t^{\prime}\right)$ is the radius at time $t$ of a bubble nucleated at time $t^{\prime}$. Applying a time derivative yields

$$
\begin{aligned}
\dot{x}(t)= & \Gamma(t)(1-x(t)) \frac{4 \pi}{3} R_{c}^{3}(t) \\
& +\int_{t_{c}}^{t} d t^{\prime} \Gamma\left(t^{\prime}\right) 4 \pi R^{2}\left(t, t^{\prime}\right) \dot{R}\left(t, t^{\prime}\right)\left(1-x\left(t^{\prime}\right)\right),
\end{aligned}
$$

where the first term corresponds to the nucleation of new bubbles while the second corresponds to the expansion of old bubbles.

The temperature evolution of the early universe plasma includes the usual adiabatic cooling term due to Hubble expansion, but now it also includes a new heating term due to the steady release of latent heat as the deconfined phase converts to the confined phase. By Eq. (A1), converting a fraction $d x$ from the deconfined to the confined phase releases $d \rho=l d x$ energy per unit volume. The released energy is absorbed in each phase with a temperature increase determined by each phase's respective specific heat, $d \rho / d T$. For now, we will focus on the large scale average temperature rather than deal with small scale temperature gradients between points near and far from the sites of latent heat release. If we assume that the portal interaction between the SM bath and dark sector leads to frequent enough interactions between the two sectors per Hubble time, then in each phase the specific heat is dominated by the many degrees of freedom of the high temperature SM bath. For example, the deconfined phase of the dark sector is found to contribute about $5 \%$ to the specific heat at the critical temperature [104]. We can then use

$$
\rho(T) \approx \frac{g_{*}(T) \pi^{2}}{30} T^{4}
$$

for both phases, where $g_{*}(T)$ is the effective number of relativistic degrees of freedom and it is $g_{*}(T) \approx 106.75$ for all temperatures of interest in our analysis. We therefore approximate both phases as having the same specific heat and find

$$
\begin{aligned}
d T & \approx l\left(\frac{d \rho(x)}{d T}\left(T_{c}\right)\right)^{-1} d x \\
& \approx 10^{-2} T_{c} d x .
\end{aligned}
$$

The total temperature evolution of the universe during the phase transition is then given by

$$
\dot{T}=-H T+10^{-2} T_{c} \dot{x},
$$

where the first term comes from the adiabatic cooling of relativistic species due to Hubble expansion. Had we considered a model in which few interactions take place between the standard model and dark sector baths per Hubble time, then the two sectors would be thermally decoupled. Note that $T$ would refer to the dark sector's temperature, which would heat relative to the SM temperature, and we would divide by the dark sector's specific heat, eliminating the factor of $10^{-2}$ in Eq. (A14).

There is an important distinction between the temperature evolution of a weakly first-order phase transition and a strongly first-order phase transition. In a weakly firstorder phase transition the latent heat in units of $T_{c}$ is typically small (see [107]), so the heating term in Eq. (A14) is negligible and the amount of supercooling does not change much due to the added latent heat. In a strongly first-order phase transition the latent heat in units of $T_{c}$ can be order one or larger [see Eq. (A2)], so $T$ can be driven back up to $T_{c}$ before the phase transition is completed. Furthermore, since $\kappa$ in Eq. (A9) scales inversely with $l^{2}$, the large value of the latent heat decreases the amount of supercooling needed to achieve efficient bubble nucleation as is seen in Eq. (A8), making it easier for the universe to reheat to a point where nucleation is negligible. In a simulation below, we show that this reheating scenario is achieved in our phase transition.

Notice also that $T$ cannot ever reheat all the way up to $T_{c}$. If it did, the critical radius would diverge and all bubbles would shrink. The second term in Eq. (A14) would change sign and become a cooling term since latent heat would be absorbed, and hence $T$ would be driven back below $T_{c}$. Instead, $\left|T-T_{c}\right|$ decreases from its maximum to an equilibrium value very close to zero at which the heating and cooling terms in Eq. (A14) nearly balance one another, as we will show below. This equilibrium phase coexistence is exactly the regime described by the Maxwell construction for first-order phase transitions (see [105], Sec. 84).

As explained in the previous section, we assume that bubbles nucleated at time $t_{0}$ are of size $R_{c}\left(T\left(t_{0}\right)\right)$, giving the initial condition $R\left(t=t_{0}, t_{0}\right)=R_{c}\left(T\left(t_{0}\right)\right)$. To determine the radius at a future time, we need an expression for the bubble wall velocity, $\dot{R}\left(t, t^{\prime}\right)$. An accurate treatment of the bubble wall velocity requires full $3+1$ dimensional 
numerical simulations of bubble dynamics during the phase transition. However, even in simplified settings, various numerical simulations have not converged on a single, definitive answer [63,67-69]. Instead, we will use a convenient, basic model of $\dot{R}\left(t, t^{\prime}\right)$. We require that critical bubbles not change their radius and that larger bubbles expand while smaller bubbles contract. To capture this behavior, we use the simple functional form

$$
\dot{R}\left(t, t_{0}\right)=v_{w}(t) \operatorname{sign}\left[R\left(t, t_{0}\right)-R_{c}(t)\right]
$$

where we define $\operatorname{sign}(0)=0$.

Determining $v_{w}(t)$ would require a better understanding of the underlying strong dynamics. Instead, we will estimate an upper bound on $v_{w}(t)$ based on thermodynamic arguments. As argued in the main text, the larger $v_{w}$ is, the less the DM relic abundance will be suppressed. Thus, we will always set $v_{w}$ to its upper bound, assuming it to be a conservative choice.

As a bubble expands, it releases latent heat near its wall, locally heating the plasma at the wall to a temperature $T_{\text {wall }}>T$ and reducing the free energy difference at the interface. Since the free energy density is minus the pressure, this local heating reduces the net pressure acting on the wall [see Eq. (A4)]. Since the degree of supercooling is so small, the wall could potentially heat up to a temperature at which the net pressure balances against the surface tension. If the wall reaches this temperature, bubble growth would no longer be thermodynamically favorable, so the wall motion would come to a halt. By an argument completely analogous to the one that led to Eq. (A3), except we evaluate Eq. (A4) at the wall temperature, we find this equilibrium wall temperature to be

$$
T_{\mathrm{wall}}^{\mathrm{eq}}=T_{c}\left(1-\frac{2 \sigma}{l R}\right)
$$

We assume that as the wall temperature approaches $T_{\text {wall }}^{\mathrm{eq}}$, its growth slows down gradually. Before the wall temperature reaches $T_{\text {wall }}^{\mathrm{eq}}$, it will have slowed down to a steady state at which the rates of wall heating and cooling cancel one another. By estimating the rates of wall heating and cooling and then setting them equal, we will determine an approximate expression for $v_{w}(t)$.

We start with the cooling rate. We will assume that $T_{\text {wall }}$ is very close to $T_{\text {wall }}^{\mathrm{eq}}$, which is, in turn, very close to $T_{c}$ since the second term in Eq. (A16) is very small compared to 1 for bubbles larger than $\Lambda^{-1}$. This assumption will lead to a faster $v_{w}$. The fractional temperature difference between points near and far from the wall is then $\left(T_{c}-T\right) / T_{c}$. We assume that the heat loss rate is given by a diffusion equation, $\dot{T}_{\text {cool }} \sim-K \nabla^{2} T$, and that the transport coefficient at $T_{c}$ is of order $K \sim \Lambda^{-1}$. If we further assume that the length scale of the density gradient is $\Lambda^{-1}$, then we find $\dot{T}_{\text {cool }} \sim-\Lambda^{2}\left(T_{c}-T\right) / T_{c}$.

Now we move on to the heating rate. We start with the energy injected per unit wall area and time, $l v_{w}$. If we assume that this energy is injected within a typical length $\Lambda^{-1}$ of the wall, then the energy injected per unit volume is $l v_{w} \Lambda$. As before, dividing by the specific heat, $d \rho / d T$, converts the energy increase into a temperature increase. Rather than assume that the specific heat is dominated by the SM degrees of freedom as we did when deriving Eq. (A13), we assume that for this process the SM degrees of freedom are irrelevant and the specific heat is dominated by the dark sector degrees of freedom, though this choice will not affect our final result. We do so because we anticipate that for most models the portal interaction between the SM and dark sector will generically take place on timescales much slower than $\Lambda^{-1}$, so it will be inefficient compared to the interactions within the dark sector. Hence, we assume that the dark degrees of freedom disperse latent heat on a fast timescale of order $\Lambda^{-1}$, and only on much longer timescales does this heat find its way into the SM degrees of freedom. ${ }^{12}$ Then, we can use $d \rho_{\mathrm{DS}} / d T \sim T^{3}$ [104], which gives a heating rate of $\dot{T}_{\text {heat }} \sim \Lambda^{2} v_{w}{ }^{13}$ The wall velocity at which both rates are in balance is then

$$
v_{w}=\left(\frac{T_{c}-T}{T_{c}}\right) .
$$

As argued in Ref. [52], we see that $v_{w}$ is suppressed by the small degree of supercooling during the phase transition.

We apply a similar argument to the wall velocity of contracting bubbles, which are smaller than the critical radius. As bubbles contract, latent heat is absorbed near the wall, decreasing the wall temperature, increasing the net pressure on the wall, and thus opposing further contraction. The contraction rate is therefore limited by the rate at which the cold wall can heat due to heat flow from the hotter surrounding plasma. Balancing the heating and cooling rates as before leads to a wall velocity given by Eq. (A17) up to a relative sign, justifying the symmetric functional form of Eq. (A15).

Again, the above expression for $v_{w}$ is, in reality, an upper bound. An expanding bubble wall cannot move any faster

\footnotetext{
${ }^{12} \mathrm{We}$ assume that the timescale over which interactions between the SM and dark sector baths exchange energy is much faster than the Hubble rate, justifying why the SM degrees of freedom are included in the heat capacity in Eq. (A13).

${ }^{13}$ If the portal interaction is actually efficient enough to keep the two sectors in equilibrium on this short timescale, then the heating rate would be suppressed by a factor of $g_{\star}$. However, the cooling rate would also be suppressed by a factor of $g_{\star}$ since $K$ is inversely proportional to the number density of interacting degrees of freedom (Sec. 3.9 of [108]). These two factors would then cancel in the expression for $v_{w}$. We therefore expect Eq. (A17) to be independent of the specific portal interaction.
} 

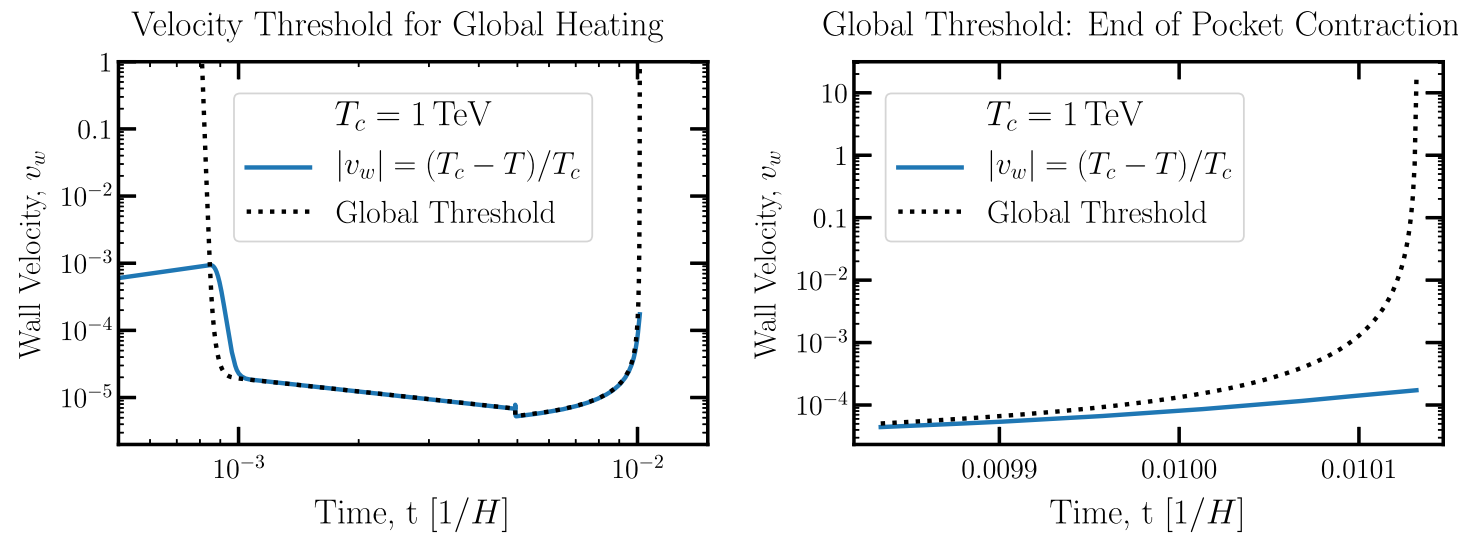

FIG. 10. Wall velocity (blue) and "global threshold" (black dashed) above which phase conversion is so fast that the universe experiences net heating. The right panel displays the same data as the left panel, but it zooms in to the very end of the phase transition when pockets have contracted significantly. The discontinuity in the middle is an artifact of our modeling. It occurs when the spectrum of bubbles, which peaks at $R_{0}$, discontinuously jumps to a delta function spectrum of pockets centered at $R_{1}$.

than Eq. (A17), at least for an extended period, because then it would locally overheat the wall. There is a similar consideration that the global rate of increase in temperature from bubble expansion should not significantly outpace the Hubble cooling, lest the whole universe be heated above $T=T_{c}$. Consequently, we see that the value of $v_{w}$ derived in Eq. (A17) evolves so that it always lies below or close to this "global threshold."

The wall velocity [Eq. (A17)] and global threshold are plotted in Fig. 10. At the start of the phase transition, when bubbles are rare and their radii are small, the global heating from bubble expansion is very small even for $v_{w} \rightarrow c$, so the global threshold velocity goes to infinity. The same is true at the end of the phase transition, when there is very little volume available for phase conversion. However, during the phase transition when the rate of phase conversion is rapid, this threshold becomes relevant.

Let us consider what happens when bubble coalescence and growth causes $v_{w}=\left(T_{c}-T\right) / T_{c}$ to exceed this global threshold (which depends on the bubble density and typical bubble radius) for the first time. If $v_{w}$ overshoots the threshold, then the universe will begin to heat up on average, thus reducing $v_{w}$. The net effect is for the degree of supercooling to evolve such that $v_{w}$ tracks the global threshold velocity. In practice, we observe that there is, at first, an abrupt drop in both $v_{w}$ and the global threshold velocity, associated with a sharp increase in the bubble number density due to nucleation; in this epoch, $v_{w}$ slightly exceeds the global threshold velocity, and this supports a fairly rapid increase in $T$. Once nucleation becomes inefficient due to the rising temperature, the global threshold velocity evolves more slowly, driven by the expansion of the largest existing bubbles. In this epoch $v_{w}$ tracks the global threshold closely and $\dot{T} \approx 0$, with a slow adiabatic increase in temperature toward $T_{c}$ driven by the slow decrease in the global threshold velocity (which requires a corresponding decrease in $v_{w}$ and hence in the degree of supercooling). We can even derive the scaling of the global threshold (and $v_{w}$ ) with $t$ during this period. By Eq. (A14), $\dot{T} \approx 0$ implies $\dot{x} \approx 100 H$. Using the fact that the spectrum of bubbles is strongly peaked at a single radius $R$ and that the nucleation of bubbles is so suppressed that the number density of bubbles is constant, we have $x=4 \pi R^{3} n_{\text {bub }} / 3$. Combining the two equations gives $d R / d t \propto R^{-2}$ so that $R=3 A\left(t-t_{0}\right)^{1 / 3}$ for some constants $A$ and $t_{0}$. Therefore, $v_{w}=d R / d t=A\left(t-t_{0}\right)^{-2 / 3}$. Indeed, we find that the slope of the line in Fig. 10 between times $0.001 / H$ and $0.005 / H$ is precisely $-2 / 3$.

The Hubble cooling can be relevant here, even though the phase transition takes place on timescales much smaller than a Hubble time, because the bubble expansion is so sensitive to the degree of supercooling; in contrast, we can freely drop e.g., density dilution terms corresponding to the Hubble expansion, as there is no comparably small density difference relevant to our calculation [see e.g., Eq. (3.2)]. After coalescence, when the heating comes from pockets of shrinking radius rather than bubbles of expanding radius, the reverse process occurs, with a slow adiabatic decrease in the equilibrium temperature due to the decreasing size of the pockets. The temperature evolution eventually switches over to the standard Hubble cooling once $v_{w}=\left(T_{c}-\right.$ $T) / T_{c}$ can no longer reach the global velocity threshold (and hence $\dot{T} \approx 0$ cannot be maintained).

With Eqs. (A11) and (A14)-(A17) in hand, we are able to simulate the first half of the phase transition. This system of equations models the initial bubble nucleation and accompanying latent heat release, and the proceeding equilibrium regime up until percolation when bubbles begin to overlap and new dynamics must be included. Our initial conditions are that the universe has supercooled a little bit, $\frac{T-T_{c}}{T_{c}}=-10^{-4}$; that the universe is fully in the 

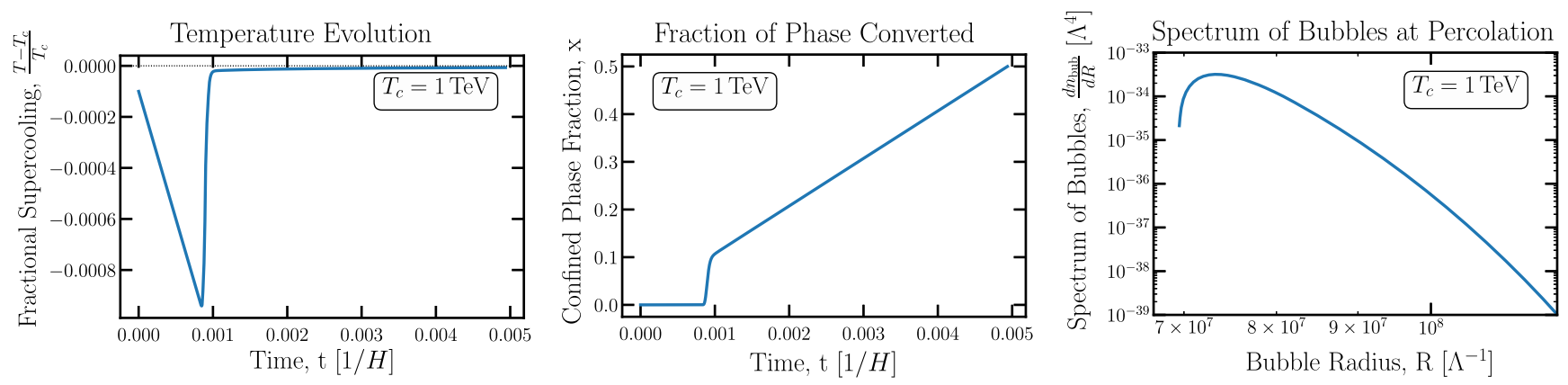

FIG. 11. Degree of supercooling as a function of time (left), confined phase fraction as a function of time (middle), and spectrum of bubble radii at percolation, when $x=1 / 2$ (right).

deconfined phase, $x=0$; and that no bubbles have nucleated yet. We evolve forward in small time steps, $\Delta t=10^{-6} / H\left(T_{c}\right)$. In each time step we nucleate $\Gamma(T(t))(1-x(t)) \Delta t$ bubbles per unit volume at radius $R_{c}(T(t))$ and add them to a list. We allow all other bubbles from previous steps to expand or contract by an amount $\dot{R}\left(t, t^{\prime}\right) \Delta t$, which only depends on the bubble size and temperature within that time step. This procedure produces bubbles with radii less than or equal to 0 , so we set such bubbles' radii to zero and remove them from our list. Additionally, many time steps result in an additional number density of bubbles that is so exponentially small that the computer sets the number density to zero. We remove these bubbles from our list, too. Each bubble nucleation and all bubble expansions increase $x$ by $\dot{x} \Delta t$ and the temperature by $\dot{T} \Delta t$ according to Eqs. (A11) and (A14). We finish our evolution once $x=\frac{1}{2}$.

Outputs of our simulation are shown in Fig. 11. The left plot shows the degree of supercooling before percolation, and the middle plot shows the fraction of the phase converted, $x$. These plots make it clear that the first half of the phase transition can be divided into three distinct stages. In the first stage, the degree of supercooling is so small that the bubble nucleation rate is too suppressed to have a significant effect on the simulation. During this period $x=0$, and the universe cools through Hubble expansion. In the second stage, the supercooling reaches a point at which nucleation becomes efficient. These nucleated bubbles quickly grow and inject heat, corresponding to the sudden jump in the temperature and $x$ a little before $t=0.001 / H$. The temperature reaches a point very close to $T_{c}$ at which nucleation of new bubbles becomes inefficient again, leading to the third stage. This stage is exactly the equilibrium phase coexistence regime described above. There are a fixed number density of large bubbles that grow and inject latent heat at such a rate so as to cancel Hubble cooling. The net effect is that $\dot{T} \approx 0$. By Eq. (A14), we have

$$
\dot{x} \approx 10^{2} \mathrm{H} \text {. }
$$

Since the temperature is constant during this stage, the Hubble rate is as well, meaning that $x$ grows linearly in time, which can be seen in our middle plot. This equation explains why the phase transition occurs over a timescale of $10^{-2} / H$. Had we assumed instead that the portal interaction between the SM bath and dark sector was very weak and led to few scatters per Hubble time, then the dark sector would not have access to the SM heat capacity, and the above factor of $10^{2}$ would be replaced by an $\mathcal{O}(1)$ factor instead.

The rightmost plot in Fig. 11 shows the spectrum of bubble radii at the time of percolation, $x=1 / 2$. The shape of this spectrum is a product of the preceding stages. The earliest bubbles were nucleated during the first stage. They had the longest time to grow but were nucleated at a time of relatively small supercooling, meaning that their number density was exponentially suppressed. So as $R$ increases away to the right of the peak of the spectrum, $d n_{\text {bub }} / d R$ decreases. Just as the second stage begins, the universe is maximally supercooled. The bubbles produced at this point are the most numerous and constitute the peak of the distribution. In the rest of the second stage, the supercooling quickly diminishes, producing an exponentially suppressed population of bubbles that are smaller than the peak radius since they have less time to grow, leading to the sharp decrease of the spectrum to the left of the peak.

We define the peak in $d n_{\text {bub }} / d R$ to occur at $R_{0}$ and empirically find that it is very well fit by the function

$R_{0} \Lambda=10^{-6} \times\left(\frac{\Lambda}{M_{\mathrm{pl}}}\right)^{-0.9}=6.7 \times 10^{7} \times\left(\frac{\Lambda}{\mathrm{TeV}}\right)^{-0.9}$.

However, $R_{0}$ is not the only relevant length scale for the bubbles. An additional length scale, $R_{1}$, emerges from the dynamics of bubble coalescence.

At percolation, bubbles frequently come into contact with one another and begin to coalesce. To model the coalescence dynamics, we borrow another argument from [52]. When two spherical bubbles of radius $R$ coalesce into one larger-radius bubble, they decrease their surface area 
and therefore reach a more energetically favorable configuration due to surface tension. The energy difference between the two configurations is $\Delta E \sim 4 \pi R^{2}\left(2-2^{\frac{2}{3}}\right) \sigma=$ $4 \pi R^{2}\left(2-2^{\frac{2}{3}}\right) \times .02 T_{c}^{3}$. This change in energy is achieved by applying a force to the mass $M$ in the bubbles over a characteristic distance $R$. So $F \sim \Delta E / R \sim M a$, where $a$ is the acceleration of the material in the bubbles. This acceleration can also be estimated as $a \sim R / t_{\text {coalesce }}^{2}$, where $t_{\text {coalesce }}$ is the coalescence timescale. If we then use that the total mass in the two bubbles is $M=2 \times \frac{4}{3} \pi R^{3} \rho_{\text {deconf }} \approx$ $\frac{8}{3} \pi R^{3} T_{c}^{4}$, where we used $\rho_{\text {deconf }}\left(T_{c}\right) \approx T_{c}^{4}$ [104], then we find

$$
\begin{aligned}
t_{\text {coalesce }} & \sim\left(\frac{M R^{2}}{\Delta E}\right)^{\frac{1}{2}} \\
& \sim 10 T_{c}^{\frac{1}{2}} R^{\frac{3}{2}}
\end{aligned}
$$

The above equation shows that small bubbles coalesce quicker than large ones. Therefore, at percolation small bubbles will quickly coalesce until they reach a size $R_{1}$ past which $t_{\text {coalesce }}$ takes longer than the timescale of percolation. From Fig. 11 we can estimate the timescale of percolation as the time it takes $x$ to change by a couple percent at around $x=\frac{1}{2}$. We find $t_{\text {perc }} \sim 10^{-3} H^{-1} \sim 10^{-3} \frac{M_{\mathrm{pl}}}{T_{c}^{2}}$ where $M_{\mathrm{pl}}=(8 \pi G)^{-1 / 2} \sim 2.4 \times 10^{18} \mathrm{GeV}$ is the reduced Planck mass and $G$ is Newton's constant. Setting the percolation timescale equal to $t_{\text {coalesce }}$ then yields the critical bubble size of

$$
R_{1} \Lambda \approx 10^{-8 / 3}\left(\frac{M_{\mathrm{pl}}}{\Lambda}\right)^{2 / 3} \approx 4 \times 10^{7}\left(\frac{\mathrm{TeV}}{\Lambda}\right)^{2 / 3},
$$

in units of $T_{c}=\Lambda$.

In Fig. 3 we plot $R_{0}$ and $R_{1}$ as a function of the confinement scale. We find that for $\Lambda \gtrsim 1 \mathrm{TeV}, R_{0}$ is less than or equal to $R_{1}$. Therefore, for this range of $\Lambda$, once our simulation finishes at $x=\frac{1}{2}$, we assume that all bubbles begin coalescing and quickly grow to radius $R_{1}$. Since $x$ does not change during this process, we assume that $T$ remains fixed, too. For $\Lambda<1 \mathrm{TeV}$, we assume that coalescence is inefficient and bubbles remain at radius $R_{0}$.

\section{Second half of the phase transition: Pocket contraction}

After percolation, most bubbles are in contact with one another. The confined regions form a web, and the deconfined regions form isolated pockets. We assume these pockets quickly attain spherical symmetry due to surface tension and also that the typical size of a bubble before percolation is equal to the typical size of a pocket after percolation, $R_{1}$.
Since all pockets are at the same initial radius, we can solve for the initial density of pockets. The number density of pockets that are all of radius $R$ satisfies

$$
1-x=\frac{4 \pi}{3} R^{3} n_{\text {pocket }} .
$$

Since $x=\frac{1}{2}$ at percolation, we have

$$
n_{\text {pocket }}=\frac{3}{8 \pi R_{1}^{3}} .
$$

We find that the degree of supercooling continues to be so small during pocket contraction that the nucleation of more bubbles is completely suppressed. Therefore, $n_{\text {pocket }}$ remains constant, and we find

$$
x=1-\frac{R^{3}}{2 R_{1}^{3}} .
$$

As before, the contraction rate of the pockets is limited due to the latent heat release near the pocket wall. However, the wall velocity estimate for pockets is slightly different than it was for bubbles. Whereas supercooling results in a net pressure outward for bubbles, supercooling results in a net pressure inward for pockets since the two phases are on opposite sides of the wall in either case. With this caveat in mind, we can repeat our argument leading to Eq. (A17).

As before, we argue that as it expands, the wall quickly heats up, approaching a threshold temperature at which pressure and surface tension are in equilibrium. This threshold now corresponds to slight superheating at the temperature $T_{c}\left(1+\frac{2 \sigma}{l R}\right)$. Before $T_{\text {wall }}$ reaches this threshold, it achieves a steady state at which heating from latent heat injection cancels against cooling from heat diffusion from the wall. Using the same arguments as before, we find that this steady state corresponds to a velocity of

$$
\frac{d R}{d t}=-\left(T_{c}-T\right) / T_{c} .
$$

Again, we use that $\frac{2 \sigma}{l R}=\frac{.03}{R \Lambda} \ll 1$, so we approximate the temperature difference between the wall and its surroundings appearing in the above equation as $T_{c}-T$, which leads to an overestimate of the wall velocity.

There is another, much more important, new effect modifying the contraction rate of a pocket: quark pressure. The density of quarks trapped within pockets increases as they are forced within ever-shrinking volumes. This pressure opposes contraction, slowing down the wall velocity relative to Eq. (A26). For now, to build intuition, we ignore the effect of quark pressure, and we consider it in the next section.

With Eqs. (A14), (A25), and (A26), we have all we need to simulate the second half of the phase transition. We use 

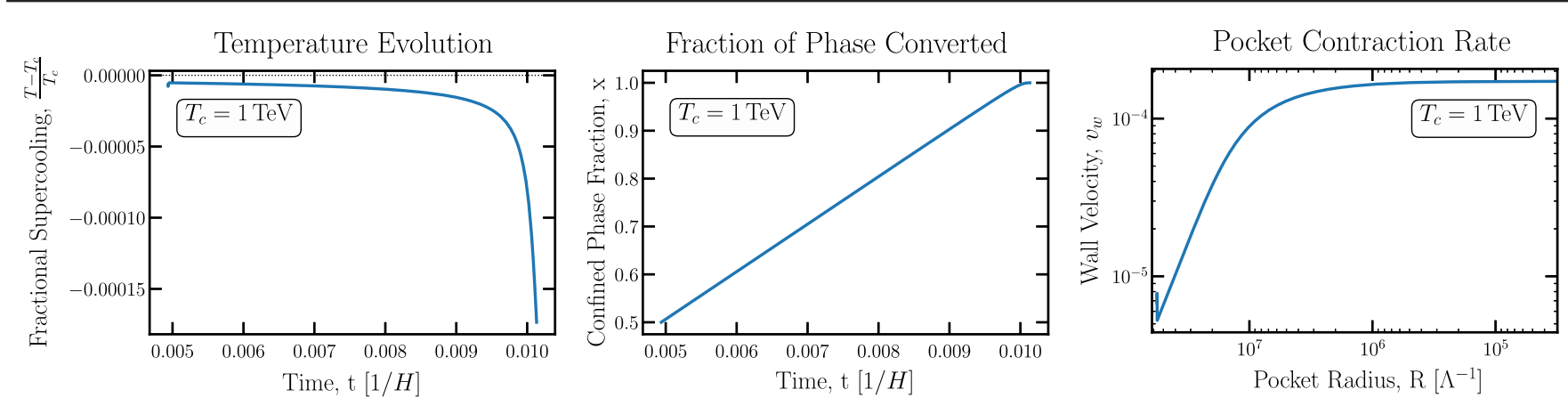

FIG. 12. Degree of supercooling as a function of time (left), confined phase fraction as a function of time (middle), and pocket wall velocity as a function of bubble radius during the second half of the phase transition (right).

the same method and parameters as we did for the first half of the phase transition. In Fig. 12 we show the results of our simulation. The first instant of the simulation features a discontinuity in the temperature (and thus $v_{w}$ ). This discontinuity results from our discontinuous change of the spectrum of bubbles from the smooth form of $d n / d R$ in the right panel of Fig. 11 to a delta function at $R_{1}$. As the pockets get smaller, their heating rate diminishes. Hubble cooling becomes relatively more important over time, leading to the steady decrease in $T$ over time. By the very end of the phase transition, the temperature evolution is purely determined by Hubble cooling.

The right panel shows the pocket wall velocity as a function of the pocket radius. As a function of $R, v_{w}$ asymptotes to a well-defined value near $2 \times 10^{-4}$ for the choice of $\Lambda$ shown in the figure. Naively, this plot seems to be at odds with the left panel since the two plots are equivalent up to a minus sign. Whereas the velocity seems to asymptote to a constant value at late times, the degree of supercooling seems to vary greatly at late times. The apparent discrepancy is a result of the different $x$-axis scales. Whereas the $x$ axis in the left panel is linearly scaled in $t$, the $x$ axis in the right panel is log scaled in $R$. The majority of the simulation takes place when the pocket radii are very large. The pocket radii are much smaller than their initial value only for a very short time at the end of the simulation, at timescales much shorter than $1 / \mathrm{H}$. In fact, the small time step $10^{-6} / H$ was chosen to resolve this smaller timescale. At such small timescales and pocket radii, very little Hubble cooling or latent heat injection takes place, leading to the plateau in $v_{w}$. Since most of the quark interactions recouple at pocket radii much smaller than $R_{1}$ (see e.g., Fig. 6), we are justified in treating $v_{w}$ as constant within the Boltzmann equations of Sec. III. ${ }^{14}$ The asymptotic $v_{w}$ value as a function of $\Lambda$ is plotted in Fig. 3 and is well fit by

\footnotetext{
${ }^{14}$ Recall that by "recoupling" we mean the point when the rates of quark interactions become large compared to the contraction rate of the pocket, so the quark density evolution is dominated by interactions.
}

$$
v_{w}\left(R \ll R_{1}\right)=0.2\left(\frac{\Lambda}{M_{\mathrm{pl}}}\right)^{0.2}=1.7 \times 10^{-4} \times\left(\frac{\Lambda}{\mathrm{TeV}}\right)^{0.2}
$$

\section{Effect of quark pressure}

Up to this point we have neglected the effect of quark pressure on the phase transition,

$$
p_{q}=n_{q} T .
$$

For the first half of the phase transition, this approximate treatment was justified. At the start of the phase transition, $p_{q}$ is suppressed compared to the gluon pressure. During the phase transition $n_{q}$ grows, so the quark pressure could potentially become large enough to affect the bubble dynamics. During the first half of the phase transition, however, the quark density, and hence $p_{q}$, grows by only a factor of 2. Including this factor of 2 enhancement, we find that the quark pressure is subdominant compared to the net gluon pressure during the bubble growth stage of the phase transition and hence can be neglected.

On the other hand, during the second half of the phase transition, the quarks are compressed much more. We find that for every point in the DM parameter space we consider, the quark pressure eventually becomes comparable to the other forces acting on the wall. Most likely, the increased quark pressure would oppose further contraction and slow $v_{w}$ down. Unfortunately, the process by which $p_{q}$ gradually grows and, in response, $v_{w}$ gradually shrinks is a nonequilibrium, strong physics problem for which we have no solution. Nevertheless, we can still use thermodynamic arguments as before to understand the possible limiting behavior of $v_{w}$.

Consider the scenario in which the enhanced quark pressure forces the pocket into a state of mechanical equilibrium. Mechanical equilibrium is achieved when the four forces on the wall-gluon pressure inside the pocket, glueball pressure outside the pocket, surface tension, and quark pressure-are in balance. At this equilibrium point, we must have 

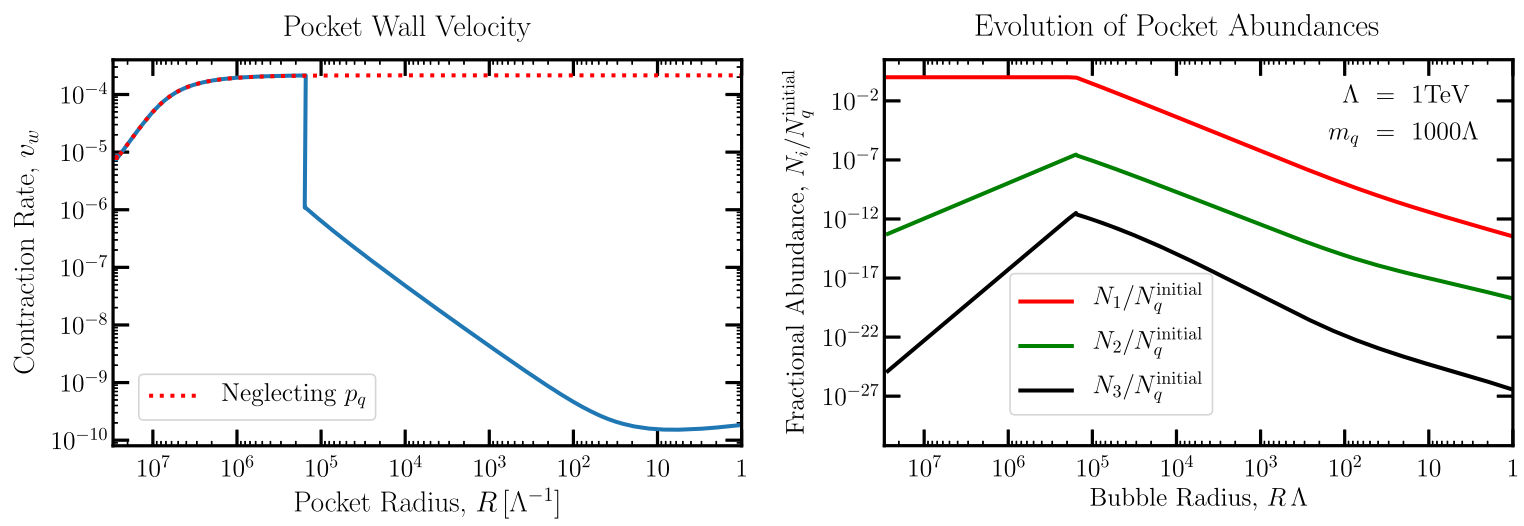

FIG. 13. Pocket wall velocity (left) and particle abundances (right) within the pocket using a model that crudely incorporates the effects of quark pressure on $v_{w}$. We choose a confinement scale of $1 \mathrm{TeV}$ and dark matter mass of $10^{3} \mathrm{TeV}$. We begin the simulation by neglecting the quark pressure, allowing us to use Eq. (A26) to determine the contraction rate (red-dotted line). Eventually, the quark pressure is so large that it is able to come into mechanical equilibrium with the other forces acting on the wall, which happens at the discontinuity near $R \Lambda=10^{5}$. At this point, we switch over to a contraction rate given by Eq. (A30), leading to the discontinuous drop in $v_{w}$ in the left panel and the sudden depletion of all particle abundances in the right panel.

$$
\begin{aligned}
0 & =d A \sigma+\left.d V\left(\sum p\right)\right|_{\text {wall }} \\
& =8 \pi \sigma R+4 \pi \Delta f\left(T_{\text {wall }}\right) R^{2}-4 \pi p_{q} R^{2} \\
& =8 \pi \sigma R+4 \pi \frac{\left(T_{c}-T_{\text {wall }}\right)}{T_{c}} l R^{2}-3 N_{q} T_{\text {wall }} R^{-1},
\end{aligned}
$$

where $\Delta f$ still refers to the confined phase minus the deconfined phase gluonic pressures. The temperatures are all evaluated at the local wall temperature, and we use $N_{q}=\frac{4 \pi}{3} R^{3} n_{q}$. If the system ever reached this equilibrium point, it would be a stable equilibrium: If $R$ were to contract, the increased quark density would oppose it; if $R$ were to expand, the surface tension would increase, the quark pressure would decrease, and the wall would absorb latent heat and increase the net gluon pressure difference, all of which oppose further expansion. After achieving mechanical equilibrium, the wall would proceed to contract adiabatically as quark annihilation decreases the quark pressure and wall cooling increases the net gluon pressure compressing the wall. By differentiating Eq. (A29) with respect to time and defining $v_{w}=-\dot{R}$, we find

$v_{w}=\frac{-3 \dot{N}_{q} T_{\text {wall }}-\left(N_{q}+\frac{4 \pi l R^{3}}{T_{c}}\right) \dot{T}_{\text {wall }}}{\left(8 \pi \sigma R+8 \pi \frac{\left(T_{c}-T_{\text {wall }}\right)}{T_{c}} l R^{2}+3 N_{q} T_{\text {wall }} R^{-1}\right)}$.

Since we do not keep track of $T_{\text {wall }}$, nor can we calculate $v_{w}$ as a function of $T_{\text {wall }}$ when the wall is out of mechanical equilibrium, we have no way of knowing when or if mechanical equilibrium is achieved.

However, to develop some intuition for the possible effects of quark pressure, we can perform a crude approximate calculation. For this calculation, we perform our pocket contraction simulation while simultaneously keeping track of the quark abundance within each step using the Boltzmann equations of Sec. III. Since the quark pressure is subdominant initially, we start with $v_{w}$ given by Eq. (A26). Eventually, there is a radius when the quark pressure is so large that it is able to oppose the combined forces of surface tension and the net gluonic pressure, even when the latter pressure is at its maximum (which is attained when the wall temperature is at its minimum, $T_{\text {wall }}=T$ ). At this radius, we say that the wall has suddenly attained mechanical equilibrium. We then switch over to a wall velocity of Eq. (A30) for the rest of the simulation. We assume that the system maintains mechanical equilibrium and $T_{\text {wall }}=T$ until the end of the simulation.

In Fig. 13 we plot the velocity and particle abundances within the pocket as functions of $R$ for this new simulation. One can see that the velocity discontinuously drops at a radius of $R \sim 10^{5} \Lambda^{-1}$ when the pocket abruptly achieves mechanical equilibrium. When this happens, further contraction is allowed only by subsequent quark annihilations; the quark depletion processes immediately recouple and dominate the density evolution due to the sharp drop in the contraction rate $v_{w} / R$. At this same radius, the baryon abundance is at a maximum, and we find that the DM relic abundance is set. Afterwards, the pocket slowly contracts, and all particle numbers are depleted until the pocket vanishes. Importantly, we find that the pocket asymmetry is saturated in this simulation [see Eq. (2.16)], and it is also saturated for every other point in the DM parameter space that we consider. Of course, this is a crude approximation-a realistic scenario could have a smoother evolution of $v_{w}$, or if the wall velocity falls sharply, this could induce plasma shock waves which modify the pocket evolution. However, this explicit example supports our intuition that turning on quark pressure will drive the system rapidly into the regime where the asymmetry is saturated, and once in this 
regime, the details of the evolution do not affect the final relic abundance.

\section{APPENDIX B: CROSS SECTIONS}

For the computation of the baryon survival factor, multiple processes are relevant. We distinguish between three classes of interactions:

(i) Annihilation process, i.e., a direct annihilation of free quarks into dark gluons: $1+(-1) \rightarrow 0+0$.

(ii) Capture processes, where a dark gluon is emitted for momentum conservation, for example: $1+1 \rightarrow 2+0$.

(iii) Rearrangement processes, where no gluon is emitted and only quark constituents exchanged, for example: $2+2 \rightarrow 3+1$.

Similar to Table I, in writing these equations we use each relic's quark number.

For the values of the annihilation and capture cross sections, explicit calculations taking into account group theory factors have been performed in $[25,26,61]$. The cross sections scale as

$$
\left\langle\sigma_{\text {ann } / \mathrm{cap}} v\right\rangle=\zeta \frac{\pi \alpha^{2}}{m_{q}^{2}} \equiv \zeta \sigma_{0},
$$

where $\zeta$ is a numerical factor that depends on the number of colors and flavors in the theory, and the coupling strength $\alpha$ is evaluated at the scale of the momentum transferred in the annihilation process, which is $m_{q}$. In addition, at low interaction energies, the bound state formation and the annihilation process experience enhancement due to nonperturbative Sommerfeld corrections. The cumulative effect of those nonperturbative effects at finite temperature can be taken into account by the effective cross section $\left\langle\sigma_{\text {eff }} v_{\text {rel }}\right\rangle$ defined in [25,26,61]. In Fig. 14 the factor $\zeta$ is shown for different annihilation and capture processes in our full set of Boltzmann equations.

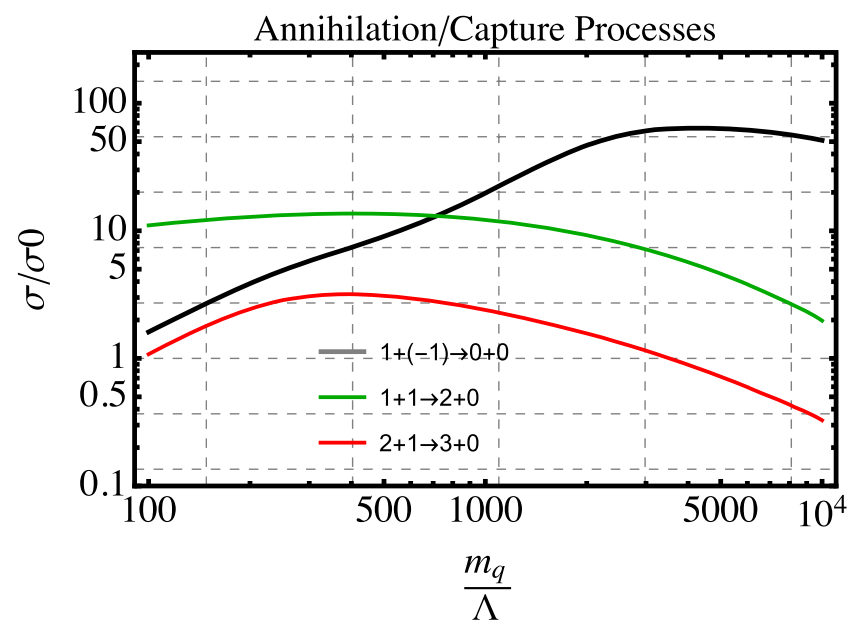

FIG. 14. Reproducing the results of $[25,26,61]$ for the $\zeta$ factor in Eq. (B1) for various annihilation or capture processes entering our full set of Boltzmann equations.
Thermal masses of the dark gluons prevent bound state formation at large temperatures, an effect that has been confirmed by additional investigations of the process at hand in a nonequilibrium field theory treatment $[61,109,110]$.

The rearrangement process is more complex and requires taking into account nonperturbative effects. Here, simulations and comparisons to hydrogen-antihydrogen annihilation have been performed in [25]. The resulting cross section scales with the area set by the Bohr radius of the colliding bound states and contains a suppression factor which becomes effective once the kinetic energy exceeds the available binding energy:

$$
\sigma_{\mathrm{RA}}=\frac{\pi R_{\mathrm{Bohr}}^{2}}{\sqrt{E_{\mathrm{kin}} / E_{\mathrm{E}_{\mathrm{B}}}}} .
$$

This results in a constant $\sigma v$ cross section, which is expected for an exothermic reaction

$$
\left\langle\sigma_{\mathrm{RA}} v\right\rangle=\frac{1}{C_{N} \alpha} \frac{\pi}{m_{q}^{2}}=\frac{\sigma_{0}}{C_{N} \alpha^{3}}
$$

with $C_{N}$ being the quadratic Casimir of the dark quark representation $\left[C_{N}=4 / 3\right.$ for quarks in the fundamental representation of $S U(N)$ ] that controls the interquark attraction in a non-Abelian theory. The overall scaling is

TABLE III. Processes and cross-section classes involved in the annihilation and baryon formation. Bound states are denoted by their baryon number. Direct annihilation takes place if multiple gluons need to be emitted in the final state. If one gluon is radiated, then perturbative capture cross-section calculations apply. In the case that the final states have no gluons that would be needed for momentum conservation, we use the geometric rearrangement cross sections discussed below. The 0 in the rearrangement processes denotes a pion $\bar{q} q$ that promptly decays to gluons. Generally, all processes above have equivalent reactions, where all particles are replaced by antiparticles. For those we assume the same cross sections, i.e., assuming $C P$ is conserved.

\begin{tabular}{llc}
\hline \hline Class & \multicolumn{1}{c}{ Process } & Cross section \\
\hline Annihilation & $1+(-1) \rightarrow 0+0$ & Fig. 14 and Eq. (B1) \\
Capture & $1+1 \rightarrow 2+0$ & \\
& $2+1 \rightarrow 3+0$ & \\
Rearrangement & $(-3)+1 \rightarrow(-1)+(-1)$ & Eq. (B3) \\
& $(-3)+1 \rightarrow(-2)+0$ & \\
& $(-2)+1 \rightarrow(-1)+0$ & \\
& $3+(-2) \rightarrow 1+0$ & \\
& $2+2 \rightarrow 3+1$ & \\
& $3+(-2) \rightarrow 2+(-1)$ & \\
& $3+(-3) \rightarrow 2+(-2)$ & \\
$2+(-2) \rightarrow 1+(-1)$ & \\
& $3+(-3) \rightarrow 1+(-1)$ & \\
& $3+(-3) \rightarrow 0+0$ & \\
$2+(-2) \rightarrow 0+0$ & \\
\hline \hline
\end{tabular}


thus $\sigma_{\mathrm{RA}} v \sim \sigma_{\text {ann } / \text { cap }} v / \alpha^{3}$, in agreement with the numerical results of [111]. This nonperturbative enhancement results from taking into account the finite size of the colliding bound states.

We summarize all the cross sections entering Eq. (3.10) in Table III. Notice that in this table, some processes involving gluons (denoted by 0 ) are listed as a rearrangement. These processes, in fact, have an intermediate step in which the quarks are rearranged and make pions $(\bar{q} q)$, which can promptly decay into gluons; see Eq. (3.1).

\section{APPENDIX C: BINDING ENERGIES}

The binding energies of several types of dark states enter the full set of Boltzmann equations. For the two quark states, exact results are available. Since we work in the limit $m_{q} \gg \Lambda_{\mathrm{DC}}$, the Coulomb potential approximation is valid. For the baryon binding energy, variational methods are needed, and numerical evaluations were performed in [25]. We focus on the case where $N=3$. The resulting binding energies are as follows: (i) Binding energy of a singlet diquark, or meson $\bar{q} q: E_{B}^{\bar{q} q}=\frac{1}{4} \alpha^{2} C_{N}^{2} m_{q}$.

(ii) Binding energy of a bound nonsinglet diquark state $q q$ in a binding configuration: $E_{B}^{q q}=\frac{1}{4} E_{B}^{\bar{q} q}$.

(iii) Binding energy of a baron $q q q$ singlet state: $E_{B}^{q q q}=0.26 \alpha^{2} C_{N}^{2} m_{q}$

Here $\alpha$ is the gauge coupling of the confining group given by Eq. (2.4). The relevant scale at which the coupling should be evaluated is the Bohr momentum $\alpha m_{q}$, which can be determined iteratively, starting from the value of $\alpha\left(m_{q}\right)$. It has been shown that the corrections due to the linear (confining) part of the potential between quarks has a negligible effect on these binding energies [see Eq. (4) of [26]].

Note that the binding energies inside and outside the pockets differ only by the subdominant contribution of the Cornell potential $\Lambda^{2} r$, evaluated at the interquark spacing $R_{b} \sim 1 /\left(\alpha m_{q}\right)$. That contribution is suppressed by $\Lambda^{2} / m_{q}^{2} \ll 1$ with respect to the ground-state binding energy, which is of the order $\alpha^{2} m_{q}$.
[1] N. Aghanim et al. (Planck Collaboration), Planck 2018 results. VI. Cosmological parameters, Astron. Astrophys. 641, A6 (2020).

[2] S. B. Gudnason, C. Kouvaris, and F. Sannino, Dark Matter from new technicolor theories, Phys. Rev. D 74, 095008 (2006).

[3] D. S. M. Alves, S. R. Behbahani, P. Schuster, and J. G. Wacker, Composite inelastic dark matter, Phys. Lett. B 692, 323 (2010).

[4] C. Kilic, T. Okui, and R. Sundrum, Vectorlike confinement at the LHC, J. High Energy Phys. 02 (2010) 018.

[5] T. Hambye and M. H. G. Tytgat, Confined hidden vector dark matter, Phys. Lett. B 683, 39 (2010).

[6] G. D. Kribs, T. S. Roy, J. Terning, and K. M. Zurek, Quirky composite dark matter, Phys. Rev. D 81, 095001 (2010).

[7] D. S. M. Alves, S. R. Behbahani, P. Schuster, and J. G. Wacker, The cosmology of composite inelastic dark matter, J. High Energy Phys. 06 (2010) 113.

[8] Y. Bai and R. J. Hill, Weakly interacting stable pions, Phys. Rev. D 82, 111701 (2010).

[9] J. L. Feng and Y. Shadmi, WIMPless dark matter from non-Abelian hidden sectors with anomaly-mediated supersymmetry breaking, Phys. Rev. D 83, 095011 (2011).

[10] R. Fok and G. D. Kribs, Chiral quirkonium decays, Phys. Rev. D 84, 035001 (2011).

[11] R. Lewis, C. Pica, and F. Sannino, Light asymmetric dark matter on the lattice: SU(2) technicolor with two fundamental flavors, Phys. Rev. D 85, 014504 (2012).

[12] M. Frigerio, A. Pomarol, F. Riva, and A. Urbano, Composite scalar dark matter, J. High Energy Phys. 07 (2012) 015.
[13] M. R. Buckley and E. T. Neil, Thermal dark matter from a confining sector, Phys. Rev. D 87, 043510 (2013).

[14] T. Appelquist et al. (Lattice Strong Dynamics (LSD) Collaboration), Lattice calculation of composite dark matter form factors, Phys. Rev. D 88, 014502 (2013).

[15] S. Bhattacharya, B. Melić, and J. Wudka, Pionic dark matter, J. High Energy Phys. 02 (2014) 115.

[16] J. M. Cline, Z. Liu, G. Moore, and W. Xue, Composite strongly interacting dark matter, Phys. Rev. D 90, 015023 (2014).

[17] K. K. Boddy, J. L. Feng, M. Kaplinghat, and T. M. P. Tait, Self-interacting dark matter from a non-Abelian hidden sector, Phys. Rev. D 89, 115017 (2014).

[18] K. K. Boddy, J. L. Feng, M. Kaplinghat, Y. Shadmi, and T. M. P. Tait, Strongly interacting dark matter: Selfinteractions and keV lines, Phys. Rev. D 90, 095016 (2014).

[19] Y. Hochberg, E. Kuflik, H. Murayama, T. Volansky, and J. G. Wacker, Model for Thermal Relic Dark Matter of Strongly Interacting Massive Particles, Phys. Rev. Lett. 115, 021301 (2015).

[20] T. Appelquist et al., Stealth dark matter: Dark scalar baryons through the Higgs portal, Phys. Rev. D 92, 075030 (2015).

[21] O. Antipin, M. Redi, A. Strumia, and E. Vigiani, Accidental composite dark matter, J. High Energy Phys. 07 (2015) 039.

[22] A. Soni and Y. Zhang, Hidden SU(N) glueball dark matter, Phys. Rev. D 93, 115025 (2016).

[23] G. D. Kribs and E. T. Neil, Review of strongly-coupled composite dark matter models and lattice simulations, Int. J. Mod. Phys. A 31, 1643004 (2016). 
[24] K. Harigaya, M. Ibe, K. Kaneta, W. Nakano, and M. Suzuki, Thermal relic dark matter beyond the unitarity limit, J. High Energy Phys. 08 (2016) 151.

[25] A. Mitridate, M. Redi, J. Smirnov, and A. Strumia, Dark matter as a weakly coupled dark baryon, J. High Energy Phys. 10 (2017) 210.

[26] V. De Luca, A. Mitridate, M. Redi, J. Smirnov, and A. Strumia, Colored dark matter, Phys. Rev. D 97, 115024 (2018).

[27] R. Contino, A. Mitridate, A. Podo, and M. Redi, Gluequark dark matter, J. High Energy Phys. 02 (2019) 187.

[28] C. Gross, A. Mitridate, M. Redi, J. Smirnov, and A. Strumia, Cosmological abundance of colored relics, Phys. Rev. D 99, 016024 (2019).

[29] V. Beylin, M. Y. Khlopov, V. Kuksa, and N. Volchanskiy, Hadronic and hadron-like physics of dark matter, Symmetry 11, 587 (2019).

[30] N. A. Dondi, F. Sannino, and J. Smirnov, Thermal history of composite dark matter, Phys. Rev. D 101, 103010 (2020).

[31] D. Buttazzo, L. Di Luzio, P. Ghorbani, C. Gross, G. Landini, A. Strumia, D. Teresi, and J.-W. Wang, Scalar gauge dynamics and dark matter, J. High Energy Phys. 01 (2020) 130.

[32] G. Landini and J.-W. Wang, Dark matter in scalar $\operatorname{Sp}(\mathcal{N})$ gauge dynamics, J. High Energy Phys. 06 (2020) 167.

[33] R. C. Brower et al. (Lattice Strong Dynamics Collaboration), Stealth dark matter confinement transition and gravitational waves, Phys. Rev. D 103, 014505 (2021).

[34] R. Contino, A. Podo, and F. Revello, Composite Dark matter from strongly-interacting chiral dynamics, J. High Energy Phys. 02 (2021) 091.

[35] V. Beylin, M. Khlopov, V. Kuksa, and N. Volchanskiy, New physics of strong interaction and Dark Universe, Universe 6, 196 (2020).

[36] S. M. Barr, Baryogenesis, sphalerons, and the cogeneration of dark matter, Phys. Rev. D 44, 3062 (1991).

[37] B. Svetitsky and L. G. Yaffe, Critical behavior at finitetemperature confinement transitions, Nucl. Phys. B210, 423 (1982).

[38] O. Kaczmarek, F. Karsch, E. Laermann, and M. Lutgemeier, Heavy quark potentials in quenched QCD at high temperature, Phys. Rev. D 62, 034021 (2000).

[39] C. Alexandrou, A. Boriç1, A. Feo, P. de Forcrand, A. Galli, F. Jegerlehner, and T. Takaishi, Deconfinement phase transition in one-flavor QCD, Phys. Rev. D 60, 034504 (1999).

[40] Y. Aoki, G. Endrodi, Z. Fodor, S. Katz, and K. Szabo, The order of the quantum chromodynamics transition predicted by the standard model of particle physics, Nature (London) 443, 675 (2006).

[41] H. Saito, S. Ejiri, S. Aoki, T. Hatsuda, K. Kanaya, Y. Maezawa, H. Ohno, and T. Umeda (WHOT-QCD Collaboration), Phase structure of finite temperature QCD in the heavy quark region, Phys. Rev. D 84, 054502 (2011); 85, 079902(E) (2012).

[42] B. Lucini, M. Teper, and U. Wenger, The high temperature phase transition in SU(N) gauge theories, J. High Energy Phys. 01 (2004) 061.
[43] B. Lucini, M. Teper, and U. Wenger, Properties of the deconfining phase transition in $\mathrm{SU}(\mathrm{N})$ gauge theories, J. High Energy Phys. 02 (2005) 033.

[44] M. J. Baker, J. Kopp, and A. J. Long, Filtered Dark Matter at a First Order Phase Transition, Phys. Rev. Lett. 125, 151102 (2020).

[45] D. Chway, T.H. Jung, and C.S. Shin, Dark matter filtering-out effect during a first-order phase transition, Phys. Rev. D 101, 095019 (2020).

[46] H. Davoudiasl and G. Mohlabeng, Getting a THUMP from a WIMP, J. High Energy Phys. 04 (2020) 177.

[47] W. Chao, X.-F. Li, and L. Wang, Filtered pseudo-scalar dark matter and gravitational waves from first order phase transition, J. Cosmol. Astropart. Phys. 06 (2021) 038.

[48] T. Konstandin and G. Servant, Cosmological consequences of nearly conformal dynamics at the TeV scale, J. Cosmol. Astropart. Phys. 12 (2011) 009.

[49] T. Hambye, A. Strumia, and D. Teresi, Super-cool dark matter, J. High Energy Phys. 08 (2018) 188.

[50] P. Baratella, A. Pomarol, and F. Rompineve, The supercooled Universe, J. High Energy Phys. 03 (2019) 100.

[51] I. Baldes, Y. Gouttenoire, and F. Sala, String fragmentation in supercooled confinement and implications for dark matter, J. High Energy Phys. 04 (2021) 278.

[52] E. Witten, Cosmic separation of phases, Phys. Rev. D 30, 272 (1984).

[53] K. Griest and M. Kamionkowski, Unitarity Limits on the Mass and Radius of Dark Matter Particles, Phys. Rev. Lett. 64, 615 (1990).

[54] B. von Harling and K. Petraki, Bound-state formation for thermal relic dark matter and unitarity, J. Cosmol. Astropart. Phys. 12 (2014) 033.

[55] J. Smirnov and J. F. Beacom, TeV-scale thermal WIMPs: Unitarity and its consequences, Phys. Rev. D 100, 043029 (2019).

[56] C. J. Moore, R. H. Cole, and C. P. L. Berry, Gravitationalwave sensitivity curves, Classical Quantum Gravity 32, 015014 (2015).

[57] P. Asadi, E. D. Kramer, E. Kuflik, G. W. Ridgway, T. R. Slatyer, and J. Smirnov, companion Letter, Accidentally Asymmetric Dark Matter, Phys. Rev. Lett. 127, 211101 (2021).

[58] C. Bonati, P. de Forcrand, M. D'Elia, O. Philipsen, and F. Sanfilippo, Constraints on the two-flavor QCD phase diagram from imaginary chemical potential, Proc. Sci., LATTICE2011 (2011) 189 [arXiv:1201.2769].

[59] G. M. Pelaggi, A. D. Plascencia, A. Salvio, F. Sannino, J. Smirnov, and A. Strumia, Asymptotically safe Standard Model extensions?, Phys. Rev. D 97, 095013 (2018).

[60] A. Patel, A flux tube model of the finite temperature deconfining transition in QCD, Nucl. Phys. B243, 411 (1984).

[61] A. Mitridate, M. Redi, J. Smirnov, and A. Strumia, Cosmological implications of dark matter bound states, J. Cosmol. Astropart. Phys. 05 (2017) 006.

[62] Particle Data Group, Review of particle physics, Prog. Theor. Exp. Phys. (2020) P083C01.

[63] S. Balaji, M. Spannowsky, and C. Tamarit, Cosmological bubble friction in local equilibrium, J. Cosmol. Astropart. Phys. 03 (2021) 051. 
[64] J. Kang and M. A. Luty, Macroscopic strings and "quirks" at colliders, J. High Energy Phys. 11 (2009) 065.

[65] J. Schwinger, On gauge invariance and vacuum polarization, Phys. Rev. 82, 664 (1951).

[66] F. Karsch, Lattice results on QCD thermodynamics, Nucl. Phys. A698, 199 (2002).

[67] J. Ignatius, K. Kajantie, H. Kurki-Suonio, and M. Laine, The growth of bubbles in cosmological phase transitions, Phys. Rev. D 49, 3854 (1994).

[68] S. J. Huber and M. Sopena, The bubble wall velocity in the minimal supersymmetric light stop scenario, Phys. Rev. D 85, 103507 (2012).

[69] J. Ellis, M. Lewicki, and J.M. No, On the maximal strength of a first-order electroweak phase transition and its gravitational wave signal, J. Cosmol. Astropart. Phys. 04 (2019) 003.

[70] M. Redi and A. Tesi, Cosmological production of dark nuclei, J. High Energy Phys. 04 (2019) 108.

[71] Y. Bai, A. J. Long, and S. Lu, Dark quark nuggets, Phys. Rev. D 99, 055047 (2019).

[72] C. Gross, G. Landini, A. Strumia, and D. Teresi, Dark Matter as dark dwarfs and other macroscopic objects: Multiverse relics?, J. High Energy Phys. 09 (2021) 033.

[73] W.-C. Huang, M. Reichert, F. Sannino, and Z.-W. Wang, Testing the dark confined landscape: From lattice to gravitational waves, Phys. Rev. D 104, 035005 (2021).

[74] D. J. Weir, Gravitational waves from a first order electroweak phase transition: A brief review, Phil. Trans. R. Soc. A 376, 20170126 (2018).

[75] C. Caprini et al., Science with the space-based interferometer eLISA. II: Gravitational waves from cosmological phase transitions, J. Cosmol. Astropart. Phys. 04 (2016) 001.

[76] N. Seto, S. Kawamura, and T. Nakamura, Possibility of Direct Measurement of the Acceleration of the Universe using $0.1-\mathrm{Hz}$ Band Laser Interferometer Gravitational Wave Antenna in Space, Phys. Rev. Lett. 87, 221103 (2001).

[77] S. Kawamura et al., The Japanese space gravitational wave antenna-DECIGO, Classical Quantum Gravity 23, S125 (2006).

[78] G. Harry, P. Fritschel, D. Shaddock, W. Folkner, and E. Phinney, Laser interferometry for the big bang observer, Classical Quantum Gravity 23, 4887 (2006); 23, 7361(E) (2006).

[79] M. Geller, A. Hook, R. Sundrum, and Y. Tsai, Primordial Anisotropies in the Gravitational Wave Background from Cosmological Phase Transitions, Phys. Rev. Lett. 121, 201303 (2018).

[80] R. H. Cyburt, J. Ellis, B. D. Fields, F. Luo, K. A. Olive, and V.C. Spanos, Nucleosynthesis constraints on a massive gravitino in neutralino dark matter scenarios, J. Cosmol. Astropart. Phys. 10 (2009) 021.

[81] M. Kawasaki, K. Kohri, T. Moroi, and Y. Takaesu, Revisiting big-bang nucleosynthesis constraints on long-lived decaying particles, Phys. Rev. D 97, 023502 (2018).

[82] L. Forestell, D. E. Morrissey, and G. White, Limits from BBN on light electromagnetic decays, J. High Energy Phys. 01 (2019) 074.
[83] C. V. Cappiello, J. I. Collar, and J.F. Beacom, New experimental constraints in a new landscape for composite dark matter, Phys. Rev. D 103, 023019 (2021).

[84] I. Goldman and S. Nussinov, Weakly interacting massive particles and neutron stars, Phys. Rev. D 40, 3221 (1989).

[85] M. Baryakhtar, J. Bramante, S. W. Li, T. Linden, and N. Raj, Dark Kinetic Heating of Neutron Stars and an Infrared Window on WIMPs, SIMPs, and Pure Higgsinos, Phys. Rev. Lett. 119, 131801 (2017).

[86] K. Van Tilburg, Stellar basins of gravitationally bound particles, Phys. Rev. D 104, 023019 (2021).

[87] R. K. Leane and J. Smirnov, Exoplanets as New Sub-GeV Dark Matter Detectors, Phys. Rev. Lett. 126, 161101 (2021).

[88] R. K. Leane, K. C. Y. Ng, and J. F. Beacom, Powerful solar signatures of long-lived dark mediators, Phys. Rev. D 95, 123016 (2017).

[89] R. K. Leane, T. Linden, P. Mukhopadhyay, and N. Toro, Celestial-body focused dark matter annihilation throughout the galaxy, Phys. Rev. D 103, 075030 (2021).

[90] M. Markevitch, A. Gonzalez, D. Clowe, A. Vikhlinin, L. David, W. Forman, C. Jones, S. Murray, and W. Tucker, Direct constraints on the dark matter self-interaction crosssection from the merging galaxy cluster 1E0657-56, Astrophys. J. 606, 819 (2004).

[91] J. L. Feng, M. Kaplinghat, H. Tu, and H.-B. Yu, Hidden charged dark matter, J. Cosmol. Astropart. Phys. 07 (2009) 004.

[92] M. R. Buckley and P. J. Fox, Dark matter self-interactions and light force carriers, Phys. Rev. D 81, 083522 (2010).

[93] M. Vogelsberger, J. Zavala, and A. Loeb, Subhaloes in self-interacting galactic dark matter haloes, Mon. Not. R. Astron. Soc. 423, 3740 (2012).

[94] J. Zavala, M. Vogelsberger, and M. G. Walker, Constraining self-interacting dark matter with the Milky Way's dwarf spheroidals, Mon. Not. R. Astron. Soc.: Lett. 431, L20 (2013).

[95] S. Tulin and H.-B. Yu, Dark matter self-interactions and small scale structure, Phys. Rep. 730, 1 (2018).

[96] K. Bondarenko, A. Sokolenko, A. Boyarsky, A. Robertson, D. Harvey, and Y. Revaz, From dwarf galaxies to galaxy clusters: Self-interacting dark matter over 7 orders of magnitude in halo mass, J. Cosmol. Astropart. Phys. 01 (2021) 043.

[97] E. O. Nadler, V. Gluscevic, K. K. Boddy, and R. H. Wechsler, Constraints on dark matter microphysics from the Milky Way satellite population, Astrophys. J. Lett. 878, L32 (2019); 897, L46(E) (2020).

[98] D. Gilman, S. Birrer, A. Nierenberg, T. Treu, X. Du, and A. Benson, Warm dark matter chills out: Constraints on the halo mass function and the free-streaming length of dark matter with eight quadruple-image strong gravitational lenses, Mon. Not. R. Astron. Soc. 491, 6077 (2020).

[99] K. Schutz, Subhalo mass function and ultralight bosonic dark matter, Phys. Rev. D 101, 123026 (2020).

[100] S. Polikanov, C. S. Sastri, G. Herrmann, K. Lutzenkirchen, M. Overbeck, N. Trautmann, A. Breskin, R. Chechik, and Z. Frankel, Search for supermassive nuclei in nature, Z. Phys. A 338, 357 (1990). 
[101] T. Celik, J. Engels, and H. Satz, The order of the deconfinement transition in SU(3) Yang-Mills theory, Phys. Lett. 125B, 411 (1983).

[102] N. H. Christ and H.-Q. Ding, Latent Heat and Critical Temperature of the Color-Deconfining Phase Transition, Phys. Rev. Lett. 60, 1367 (1988).

[103] B. Lucini, P. de Forcrand, and M. Vettorazzo, Measuring interface tensions in $4 \mathrm{~d} \mathrm{SU}(\mathrm{N})$ lattice gauge theories, Nucl. Phys. B140, 647 (2005).

[104] L. Giusti and M. Pepe, Equation of state of the SU(3) Yang-Mills theory: A precise determination from a moving frame, Phys. Lett. B 769, 385 (2017).

[105] L. Landau and E. Lifshitz, Statistical Physics, Part 1, Course of Theoretical Physics (Pergamon Press, Oxford, 1980), Vol. 5.

[106] L. Landau and E. Lifshitz, Physical Kinetics, Course of Theoretical Physics (Pergamon Press, Oxford, 1981), Vol. 10.
[107] V. A. Rubakov and D. S. Gorbunov, Introduction to the Theory of the Early Universe: Hot Big Bang Theory (World Scientific, Singapore, 2017).

[108] M. Kardar, Statistical Physics of Particles (Cambridge University Press, Cambridge, England, 2007).

[109] T. Binder, K. Mukaida, and K. Petraki, Rapid Bound-State Formation of Dark Matter in the Early Universe, Phys. Rev. Lett. 124, 161102 (2020).

[110] T. Binder, B. Blobel, J. Harz, and K. Mukaida, Dark matter bound-state formation at higher order: A non-equilibrium quantum field theory approach, J. High Energy Phys. 09 (2020) 086.

[111] M. Geller, S. Iwamoto, G. Lee, Y. Shadmi, and O. Telem, Dark quarkonium formation in the early Universe, J. High Energy Phys. 06 (2018) 135.

[112] See https://github.com/gridgway/ConfiningPT_Bubbles. 